\title{
Structure and stability of non-symmetric Burgers vortices
}

\author{
By AURELIUS PROCHAZKA AND D. I. PULLIN \\ Graduate Aeronautical Laboratories, California Institute of Technology, \\ Pasadena, CA 91125, USA
}

(Received 2 February 1997 and in revised form 2 December 1997)

We investigate, numerically and analytically, the structure and stability of steady and quasi-steady solutions of the Navier-Stokes equations corresponding to stretched vortices embedded in a uniform non-symmetric straining field, $(\alpha x, \beta y, \gamma z), \alpha+\beta+\gamma=0$, one principal axis of extensional strain of which is aligned with the vorticity. These are known as non-symmetric Burgers vortices (Robinson \& Saffman 1984). We consider vortex Reynolds numbers $R=\Gamma /(2 \pi v)$ where $\Gamma$ is the vortex circulation and $v$ the kinematic viscosity, in the range $R=1-10^{4}$, and a broad range of strain ratios $\lambda=(\beta-\alpha) /(\beta+\alpha)$ including $\lambda>1$, and in some cases $\lambda \gg 1$. A pseudo-spectral method is used to obtain numerical solutions corresponding to steady and quasi-steady vortex states over our whole $(R, \lambda)$ parameter space including $\lambda>1$, where arguments proposed by Moffatt, Kida \& Ohkitani (1994) demonstrate the non-existence of strictly steady solutions. When $\lambda \gg 1, R \gg 1$ and $\varepsilon \equiv \lambda / R \ll 1$, we find an accurate asymptotic form for the vorticity in a region $1<r /(2 v / \gamma)^{1 / 2} \leqslant \varepsilon^{-1 / 2}$, giving very good agreement with our numerical solutions. This suggests the existence of an extended region where the exponentially small vorticity is confined to a nearly cat's-eye-shaped region of the almost two-dimensional flow, and takes a constant value nearly equal to $\Gamma \gamma /(4 \pi v) \exp [-1 /(2 e \varepsilon)]$ on bounding streamlines. This allows an estimate of the leakage rate of circulation to infinity as $\partial \Gamma / \partial t=(0.48475 / 4 \pi) \gamma \varepsilon^{-1} \Gamma \exp (-1 / 2 e \varepsilon)$ with corresponding exponentially slow decay of the vortex when $\lambda>1$. An iterative technique based on the power method is used to estimate the largest eigenvalues for the non-symmetric case $\lambda>0$. Stability is found for $0 \leqslant \lambda \leqslant 1$, and a neutrally convective mode of instability is found and analysed for $\lambda>1$. Our general conclusion is that the generalized non-symmetric Burgers vortex is unconditionally stable to twodimensional disturbances for all $R, 0 \leqslant \lambda \leqslant 1$, and that when $\lambda>1$, the vortex will decay only through exponentially slow leakage of vorticity, indicating extreme robustness in this case.

\section{Introduction and motivation}

\subsection{Burgers vortex}

Two important dynamical mechanisms active in the fine scales of turbulence are known to be the intensification of vorticity through vortex stretching and the dissipation of energy produced in regions of large velocity gradients. This was first noted by Taylor (1938) and later, Burgers (1948) found an exact solution to the Navier-Stokes equations for a constant-density fluid that modelled these processes. Burgers' solution may be obtained by first decomposing the velocity field in Cartesian coordinates $(x, y, z)$ into an irrotational part corresponding to pure strain $\boldsymbol{u}_{s}=(\alpha x, \beta y, \gamma z)$ with 
$\alpha+\beta+\gamma=0$, and a rotational part confined to the $(x, y)$-plane, $\boldsymbol{u}_{\omega}=(u, v, 0)$. The only non-zero component of the vorticity, $\omega=\nabla \times \boldsymbol{u}$ is that in the $z$-direction, $\omega=(0,0, \omega)$, and the relevant vorticity equation then reduces to

$$
\begin{gathered}
\frac{\partial \omega}{\partial t}+(\alpha x+u) \frac{\partial \omega}{\partial x}+(\beta y+v) \frac{\partial \omega}{\partial y}=\gamma \omega+v \nabla^{2} \omega, \\
\nabla^{2} \psi=-\omega, \quad u=\frac{\partial \psi}{\partial y}, \quad v=-\frac{\partial \psi}{\partial x},
\end{gathered}
$$

where $\psi$ is the stream function. For the case of axisymmetric strain, $\alpha=\beta=-\gamma / 2$, $\gamma>0$, a steady solution of (1.1)-(1.2) is

$$
\omega=\frac{\gamma \Gamma}{4 \pi v} \mathrm{e}^{-\gamma\left(x^{2}+y^{2}\right) / 4 v}
$$

which induces the azimuthal velocity,

$$
\hat{u}_{\theta_{0}}=\frac{\Gamma}{2 \pi r}\left(1-\mathrm{e}^{-\gamma r^{2} / 4 v}\right),
$$

where $\Gamma$ is the total circulation. We will refer to this solution as the axisymmetric Burgers vortex with the Reynolds number defined as $R=\Gamma / 2 \pi v$. For the case of plane strain $\alpha=-\gamma, \beta=0, \gamma>0$, a steady flow solution of (1.1)-(1.2) is

$$
\omega=\omega_{0} \mathrm{e}^{-\gamma x^{2} / 2 v},
$$

where $\omega_{0}$ is the maximum vorticity. This solution is often referred to as the Burgers vortex layer.

\subsubsection{Relevance to turbulence}

Burgers vortices have been used to model various features of turbulence including the energy spectrum in the range of dissipation wavenumbers (Townsend 1951) and the nearly streamwise vortices which form in the braid region of the temporal mixing layer (Lin \& Corcos 1984). Interest in the properties of the Burgers vortex intensified following large-scale numerical simulations of turbulence, for example Kerr (1985), Vincent \& Meneguzzi (1991), She, Jackson \& Orszag (1990), Ashurst et al. (1987), Ruetsch \& Maxey (1991) and others, which indicated that regions of high vorticity seemed to 'self-organize' into tube-like structures. Ashurst et al. (1987) demonstrated a moderate correlation between the direction of the vorticity vector and that of the strain eigenvector corresponding to the intermediate eigenvalue and this, together with the tube observation, revived interest in the Townsend-Burgers model of the fine scales. Whilst the structure of the Burgers vortex may be too simple to explain the principal characteristics of the probability distribution of the longitudinal velocity gradients (Saffman \& Pullin 1996), the numerical experiments of Jiménez et al. (1993) do provide evidence that the structure of the most intense vorticity, which occupies a small fraction of the fluid volume and provides a small fraction of the dissipation, but which may be responsible for the tails of the velocity-gradient probability density functions, closely resembles Burgers vortices. Furthermore, Jiménez \& Wray (1994) note that whenever vortices are strongly stretched, the radius approaches the Burgers limit, and that the cross-section of these long coherent vortices will be elliptical in nature.

\subsubsection{Stability}

Leibovich \& Holmes (1981) addressed the global stability of the axisymmetric Burgers vortex using an energy method but found that there existed no finite 


$$
\begin{array}{ccl}
\lambda=0, & \alpha=\beta=-\gamma / 2, & \text { axisymmetric axial strain } \\
0<\lambda<1, & \alpha<\beta<0, & \text { non-symmetric axial strain } \\
\lambda=1, & \alpha=-\gamma, \beta=0, & \text { plane strain } \\
1<\lambda<3, & \beta>0, \beta<\gamma, & \text { biaxial strain } \\
\lambda=3, & \alpha=-2 \gamma, \beta=\gamma, & \text { axisymmetric biaxial strain } \\
\lambda>3, & \beta>\gamma, & \text { extreme biaxial strain } \\
\lambda \rightarrow \infty, & -\alpha \approx \beta \gg \gamma, & \text { two-dimensional strain limit }
\end{array}
$$

TABLE 1. Values of $\lambda, \alpha$, and $\beta$ in various classes of straining fields.

critical viscosity at which the vortex became unstable. They noted that this did not indicate stability to all perturbations. Robinson \& Saffman (1984, hereafter referred to as RS84), using perturbation methods, solved the corresponding linearized stability problem through a series expansion in $R$, finding the Burgers vortex to be linearly stable for small $R$. Prochazka \& Pullin (1995) extended this result to show that the axisymmetric Burgers vortex remains stable to infinitesimal two-dimensional disturbances for $R=1-10^{4}$.

\subsection{Stretched vortices in non-symmetric strain}

\subsubsection{Symmetry considerations}

Direct numerical simulations not only suggest the existence of vortex structures, but also the tendency of these structures to persist over long periods of time, even when their length reaches the integral scale of the flow (Vincent \& Meneguzzi 1991). Flow visualization by Douady, Coudet \& Brachet (1991) of homogeneous turbulence has also shown the existence of intense vortex tubes. In all these cases it seems reasonable to suppose that the vortex tubes were present in regions where the strain was not perfectly axially symmetric, suggesting the relevance of non-symmetric Burgers vortices. Here it is convenient to consider, without loss of generality, cases where $\alpha<0 \leqslant \gamma, \beta \geqslant \alpha$ and define the field in terms of a single parameter, the strain ratio, $\lambda$, where

$$
\alpha=-\frac{1}{2}(1+\lambda) \gamma, \quad \beta=-\frac{1}{2}(1-\lambda) \gamma, \quad \lambda=\frac{\alpha-\beta}{\alpha+\beta} .
$$

The strain ratio is non-negative and uniquely defines the strain into classifications given in table 1 . For $\lambda>3$, it should be noted that the $z$-direction is no longer the direction of maximum extensional strain.

\subsubsection{Vortex structure}

RS84 first proposed the generalized or non-symmetric Burgers vortex corresponding to strain geometries intermediate between axisymmetric and plane strain. They found numerically steady solutions at Reynolds numbers up to 100 and strain ratios $0.25 \leqslant \lambda \leqslant 0.75$. They showed that increased non-symmetry of the strain tended to increase the ellipticity of the vortex while increasing the circulation decreased the ellipticity and rotated the vortex counterclockwise to a limit where the vortex was aligned $45^{\circ}$ to the axes of the strain. Kida \& Ohkitani (1992), in a study of the spatiotemporal intermittency of developed turbulence, found that vorticity tended to be concentrated in long thin tube-like regions that resemble non-symmetric Burgers vortices in structure. Moffatt, Kida \& Ohkitani $(1994$, which we will refer to as MKO94), developed a large Reynolds number asymptotic theory of stretched vortices in non-symmetric straining fields. The structure of the vortices for the small parameter, 
$\lambda / R$, was given as a correction to that of a Burgers vortex. Jiménez, Moffatt \& Vasco (1996) showed the asymptotic results from MKO94 to be valid in a larger, elliptical region. Furthermore, they provide evidence of vortices with elliptical structure in a numerical simulation of decaying two-dimensional turbulence.

\subsubsection{Stability}

No comprehensive analysis of the stability of non-symmetric Burgers vortices has been presented to date. Buntine \& Pullin (1989) calculated several examples of the relaxation of an initial vorticity distribution towards a non-symmetric state, indicating at least some measure of the stability to two-dimensional disturbances for $\lambda<1$. MKO94 argued that the stretched vortices can survive for long times even when two of the principal rates of strain become positive. From a far-field analysis of the vorticity, they indicate that for biaxial strain, vorticity away from the core of the vortex will behave like a passive scalar and be convected to infinity, therefore eliminating the possibility of a true steady solution in the region $\lambda>1$. They further point out that there will be a tilt instability present for $\lambda>3$. This corresponds to $\beta>\gamma$ in which case the $y$-axis will become the direction of maximum extensional strain. A small perturbation of the vortex away from alignment with the $z$-axis, while maintaining the rectilinear vorticity, will be followed by a rotation of the vortex-axis on a time scale $O(\beta-\gamma)^{-1}$. There will be eventual re-alignment with the $y$-axis, after which a situation corresponding to $1<\lambda<3$ will prevail. Indeed this instability would be present for nominally two-dimensional compact vortex flows where a linear $(x, y)$-plane strain field is present, and when tilting disturbances in three dimensions are admitted.

\subsection{Outline}

We begin in $\$ 2$ by utilizing an extension of the pseudo-spectral method of RS84 to calculate solutions of the Navier-Stokes equations corresponding to non-symmetric Burgers vortices, over a wide range of $(R, \lambda)$ space. When $R$ is large and $\lambda>1$ we have no difficulty in obtaining apparently steady solutions with vorticity at the level of machine precision far from the vortex core. When $\lambda \gg 1$, our numerical results suggest a method for obtaining an approximate analytical form for the vorticity field outside the region studied by MKO94 but confined by the strain to a cat's-eye-shaped region of nearly two-dimensional flow. This approximation, developed in $\S 3$, confirms the exponentially small vorticity away from the core and also provides a means of estimating the exponentially slow leakage and convection to infinity of vorticity from the confinement zone. Our estimate of the decay rate of the circulation differs substantially from MKO94.

The two-dimensional stability of non-symmetric Burgers vortices is studied in §4. A Lagrangian convectively neutral mode corresponding to an arbitrary bulk displacement of a general vorticity distribution embedded in a general linear velocity field is identified. When applied to the steady generalized Burgers vortex, this implies a convectively neutral stability which convects the vortex to infinity without change of form when $\lambda>1$. The normal mode stability of the numerically obtained nonsymmetric steady vortex solutions is studied in $\$ 5$ using an extension of the power method. For the cases studied at $R=1,10,100$ and 1000, when $0<\lambda<1$ (non-symmetric axial strain), the largest eigenvalue always corresponds to the abovementioned convectively neutral instability mode which, for this range of $\lambda$, convects small bulk displacement of the vorticity field into the undisturbed centre of vorticity. This implies stability of the non-symmetric Burgers vortices for $0 \leqslant \lambda<1$, for all $R$. 
When $\lambda>1$, the convectively neutral mode is also found and the algebraically second largest eigenvalue is zero to the accuracy of our calculation.

\section{Structure of non-symmetric Burgers vortices}

\subsection{Basic equations}

Unless otherwise noted, all quantities are henceforth non-dimensionalized using the length and time scales $(2 v / \gamma)^{1 / 2}$ and $2 / \gamma$ respectively. In addition, in order to fix the circulation in the scaled form, the magnitude of the non-dimensional vorticity and streamfunction are scaled on the Reynolds number. Equations (1.1)-(1.2) become

$$
\begin{gathered}
\frac{\partial \omega}{\partial t}=\frac{\partial^{2} \omega}{\partial x^{2}}+\frac{\partial^{2} \omega}{\partial y^{2}}+[(1+\lambda) x-R u] \frac{\partial \omega}{\partial x}+[(1-\lambda) y-R v] \frac{\partial \omega}{\partial y}+2 \omega, \\
\nabla^{2} \psi=-\omega, \quad u=\frac{\partial \psi}{\partial y}, \quad v=-\frac{\partial \psi}{\partial x},
\end{gathered}
$$

where $\omega, \psi, u, v, x, y$, and $t$, now denote dimensionless variables without change of notation. This equation has three notable solutions. First, there is the axisymmetric Burgers vortex solution, valid for all $R$ at $\lambda=0$,

$$
\omega=\mathrm{e}^{-\left(x^{2}+y^{2}\right) / 2} .
$$

Second, we have,

$$
\omega=\left(1-\lambda^{2}\right)^{1 / 2} \mathrm{e}^{-\left((1+\lambda) x^{2}+(1-\lambda) y^{2}\right) / 2},
$$

which is valid for all $\lambda$ at $R=0$, and third, for plane strain, there exists the Burgers vortex-layer solution,

$$
\omega=\omega_{0} \mathrm{e}^{-x^{2}},
$$

which holds for $\lambda=1$ for all $\omega_{0}$, but does not correspond to a confined vorticity distribution and will not be studied here, but is referred to in $\S 3$.

\subsection{Numerical method}

\subsubsection{Pseudo-spectral method}

Here we investigate numerically steady solutions to (2.1) for general $\lambda>0$. We assume that $\omega(x, y)$ decays exponentially when $r=\left(x^{2}+y^{2}\right)^{1 / 2} \rightarrow \infty$ independent of direction. This allows use of a collocation method to approximate the vorticity with the double series of sinc functions in $x$ and $y$ (see RS84),

$$
\omega(x, y)=\sum_{k=-M}^{M} \sum_{l=-N}^{N} \omega_{k l} S\left(k, h_{x} ; x\right) S\left(l, h_{y} ; y\right),
$$

where

$$
S(j, h ; \zeta)=\frac{\sin [(\pi / h)(\zeta-j h)]}{(\pi / h)(\zeta-j h)}
$$

in a rectangular domain defined by collocation points $\left(x_{i}, y_{j}\right)=\left(i h_{x}, j h_{y}\right), i=-M \ldots M$, $j=-N \ldots N$, where $\omega_{k l}$ are coefficients to be determined and $M, N$ are specified series-truncation parameters. Integration and differentiation of the sinc function can 
be approximated using

$$
\left.\begin{array}{rl}
\int_{-\infty}^{+\infty} \omega(x, y) \mathrm{d} x & =h_{x} \sum_{k=-M}^{M} \sum_{l=-N}^{N} \omega_{k l} S\left(l, h_{y} ; y\right), \\
\frac{\partial^{n} \omega}{\partial x^{n}}(x, y) & =\sum_{k=-M}^{M} \sum_{l=-N}^{N}\left(h_{x}^{-n} \sum_{p=-M}^{M} \delta_{p k}^{(n)} \omega_{p l}\right) S\left(k, h_{x} ; x\right) S\left(l, h_{y} ; y\right) .
\end{array}\right\}
$$

We will only need $n=1,2$, for which

$$
\delta_{m n}^{(1)}=\frac{(-1)^{n-m}}{n-m}\left(1-\delta_{n m}\right), \quad \delta_{n m}^{(2)}=-\frac{1}{3} \pi^{2} \delta_{n m}-\frac{2(-1)^{n-m}}{(n-m)^{2}}\left(1-\delta_{n m}\right) .
$$

The Poisson equation (2.2) may be solved in terms of the Fourier transform of the vorticity $\hat{\omega}$, from which the velocity components may be expressed as

$$
\begin{gathered}
u=-\frac{\partial \psi}{\partial y}=\frac{-\mathrm{i}}{(2 \pi)^{2}} \int_{-\infty}^{\infty} \int_{-\infty}^{\infty} \frac{\eta \hat{\omega}}{\xi^{2}+\eta^{2}} \mathrm{e}^{-\mathrm{i}(\xi x+\eta y)} \mathrm{d} \xi \mathrm{d} \eta, \\
v=\frac{\partial \psi}{\partial x}=\frac{\mathrm{i}}{(2 \pi)^{2}} \int_{-\infty}^{\infty} \int_{-\infty}^{\infty} \frac{\xi \hat{\omega}}{\xi^{2}+\eta^{2}} \mathrm{e}^{-\mathrm{i}(\xi x+\eta y)} \mathrm{d} \xi \mathrm{d} \eta .
\end{gathered}
$$

Then, taking the Fourier transform of (2.6) and using the result that the Fourier transform of the sinc-function (2.7) is

$$
\hat{S}(j, h ; \zeta)=h \mathrm{e}^{\mathrm{i}(\zeta j h)}[H(\zeta+\pi / h)-H(\zeta-\pi / h)],
$$

where $H$ is the Heaviside function, gives expressions for the velocities at each of the collocation points in terms of the coefficients $\omega_{k l}$ in (2.6) as

$$
u_{i j}=-\frac{h_{x}}{\pi} \sum_{k=-M}^{M} \sum_{l=-N}^{N} I_{j-l, i-k}^{(1)} \omega_{k l}, \quad v_{i j}=\frac{h_{y}}{\pi} \sum_{k=-M}^{M} \sum_{l=-N}^{N} I_{i-k, j-l}^{(2)} \omega_{k l},
$$

and where

$$
\begin{aligned}
& I_{m, n}^{(1)}=\int_{0}^{1} \int_{0}^{1} \frac{\sigma \eta}{\sigma^{2} \xi^{2}+\eta^{2}} \sin ((j-l) \pi \eta) \cos ((i-k) \pi \xi) \mathrm{d} \xi \mathrm{d} \eta \\
& I_{m, n}^{(2)}=\int_{0}^{1} \int_{0}^{1} \frac{\sigma \xi}{\sigma^{2} \xi^{2}+\eta^{2}} \sin ((i-k) \pi \xi) \cos ((j-l) \pi \eta) \mathrm{d} \xi \mathrm{d} \eta
\end{aligned}
$$

where $\sigma=h_{y} / h_{x}$. Due to the difficulty in evaluating these double integrals, it is more efficient to calculate $I_{m, n}$ numerically using

$$
\begin{aligned}
I_{m, n}^{(1)}= & \int_{0}^{1} \frac{\sigma}{1+\sigma^{2} x^{2}}\left[\frac{\sin ^{2}[(m \pi+n \pi x) / 2]}{m \pi+n \pi x}+\frac{\sin ^{2}[(m \pi-n \pi x) / 2]}{m \pi-n \pi x}\right] \mathrm{d} x \\
& +\int_{0}^{1} \frac{\sigma}{\sigma^{2}+x^{2}}\left[\frac{\sin ^{2}[(m \pi x+n \pi) / 2]}{m \pi x+n \pi}+\frac{\sin ^{2}[(m \pi x-n \pi) / 2]}{m \pi x-n \pi}\right] \mathrm{d} x, \\
I_{m, n}^{(2)}= & \int_{0}^{1} \frac{\sigma}{\sigma^{2}+x^{2}}\left[\frac{\sin ^{2}[(m \pi+n \pi x) / 2]}{m \pi+n \pi x}+\frac{\sin ^{2}[(m \pi-n \pi x) / 2]}{m \pi-n \pi x}\right] \mathrm{d} x \\
& +\int_{0}^{1} \frac{\sigma}{1+\sigma^{2} x^{2}}\left[\frac{\sin ^{2}[(m \pi x+n \pi) / 2]}{m \pi x+n \pi}+\frac{\sin ^{2}[(m \pi x-n \pi) / 2]}{m \pi x-n \pi}\right] \mathrm{d} x .
\end{aligned}
$$


Then, by inserting (2.6) into (2.1) and using (2.8), we obtain

$$
\begin{aligned}
F_{i j}= & \frac{1}{h_{x}^{2}} \sum_{k=-M}^{M} \delta_{k i}^{(2)} \omega_{k j}+\frac{1}{h_{y}^{2}} \sum_{l=-N}^{N} \delta_{l j}^{(2)} \omega_{i l}+2 \omega_{i j} \\
& +\left[(1+\epsilon) i-\frac{R}{h_{x}} u_{i j}\right] \sum_{k=-M}^{M} \delta_{k i}^{(1)} \omega_{k j}+\left[(1-\epsilon) j-\frac{R}{h_{y}} u_{i j}\right] \sum_{l=-N}^{N} \delta_{l j}^{(1)} \omega_{i l}, \\
= & 0, \quad i=-M \ldots M, j=-N \ldots N,
\end{aligned}
$$

where $F_{i j}$ is the right-hand side of $(2.1)$ evaluated at the $(i, j)$ th collocation point. Equations $(2.18)$ are $(2 M+1) \times(2 N+1)$ equations for the $(2 M+1) \times(2 N+1)$ unknowns $\omega_{k l}$. Two constraints are applied to this equation: first, we fix the total circulation and second, we fix the centroid of vorticity in accordance with RS84. The circulation is fixed at $2 \pi$ by adding the term $\rho(i, j)\left(h_{x} h_{y} \sum_{k=-M}^{M} \sum_{l=-N}^{N} \omega_{k l}-2 \pi\right)$ to (2.18) where $\rho(i, j)$ is a random number function that decouples the last term from the other equations. Second, since the equations are invariant under a $180^{\circ}$ rotation, $F_{i j}=F_{-i,-j}$, we can fix the centroid of vorticity at the origin and also reduce the number of unknowns to $(2 M+1) N+M+1$.

\subsubsection{Accuracy}

With $M$ and $N$ fixed, this system is solved using the Newton-Raphson method where the Jacobian is $J_{i j k l}=\partial F_{i j} / \partial \omega_{k l}$. Euler continuation in $\lambda$ starting from the axisymmetric Burgers vortex is used to expedite results, but this is not necessary for convergence, which is defined by $\left|J_{i j k l}\left(\omega_{k l}^{\text {new }}-\omega_{k l}^{\text {old }}\right)\right|_{\max }<10^{-12}$ and at the boundary $|\omega|_{\max }<10^{-5}$. Second-order convergence is always obtained within a few iterations. Given that the Jacobian contains $((2 M+1) N+M+1)^{2}$ elements, we were limited by storage capacity to a maximum square domain of $M, N=50$, rather than by a CPU time constraint.

In order to determine the best possible values for the basis spacing $h_{x}$ and $h_{y}$, the code was tested using the axisymmetric case and it was found, by trial and error, that both the error from the Newton-Raphson method and the maximum value of the vorticity at the boundary are minimized by using $h_{x}=(\pi / M)^{1 / 2}$ and $h_{y}=(\pi / N)^{1 / 2}$, leading to a domain size of $(\pi M)^{1 / 2} \times(\pi N)^{1 / 2}$. Since the Burgers vortex is axisymmetric, the best domain shape corresponds to a square, $M=N$, but choice of domain aspect ratio depends quite strongly on the shape of the function to be approximated. At low Reynolds numbers, strain tends to elongate the vortex, therefore a rectangular domain can be used for greater accuracy. At higher Reynolds numbers, the vortex tends to be less elliptical, thus $M=N$ is used as before, but since the vortex aligns itself at an angle to the strain, domain size efficiency can be maximized by allowing strain to be rotated through an angle, $\phi$, where the velocities are

$$
\begin{aligned}
& u=\alpha x \cos ^{2} \phi+\beta x \sin ^{2} \phi+\frac{\beta-\alpha}{2} y \sin 2 \phi, \\
& v=\beta y \cos ^{2} \phi+\alpha y \sin ^{2} \phi+\frac{\beta-\alpha}{2} x \sin 2 \phi,
\end{aligned}
$$

choosing $\phi$ to align the major axis of the vortex with the diagonal of the domain.

\subsection{Results}

Since exact solutions (2.3)-(2.4) are known for either $R=0$ or $\lambda=0$, the $R, \lambda$ parameter space for Reynolds numbers $1<R<10^{4}$ and $0.2<\lambda<150$ is investigated. 


$\begin{array}{ccccc}\lambda & \omega(r=0)(R=1) & \left.\omega_{\text {max }}\right|_{\text {boundary }}(R=1) & \omega(r=0)(R=10) & \left.\omega_{\text {max }}\right|_{\text {boundary }}(R=10) \\ 0.2 & 0.9802 & 2.23 \times 10^{-16} & 0.9932 & 6.62 \times 10^{-13} \\ 0.4 & 0.9179 & 9.27 \times 10^{-16} & 0.9711 & 2.35 \times 10^{-11} \\ 0.6 & 0.8025 & 8.66 \times 10^{-16} & 0.9268 & 1.15 \times 10^{-10} \\ 0.8 & 0.6027 & 1.00 \times 10^{-10} & 0.8332 & 1.77 \times 10^{-10}\end{array}$

TABLE 2 . Vorticity values at the core and the boundary $R=1, R=10$ with $M, N=40, \phi=\pi / 4$.
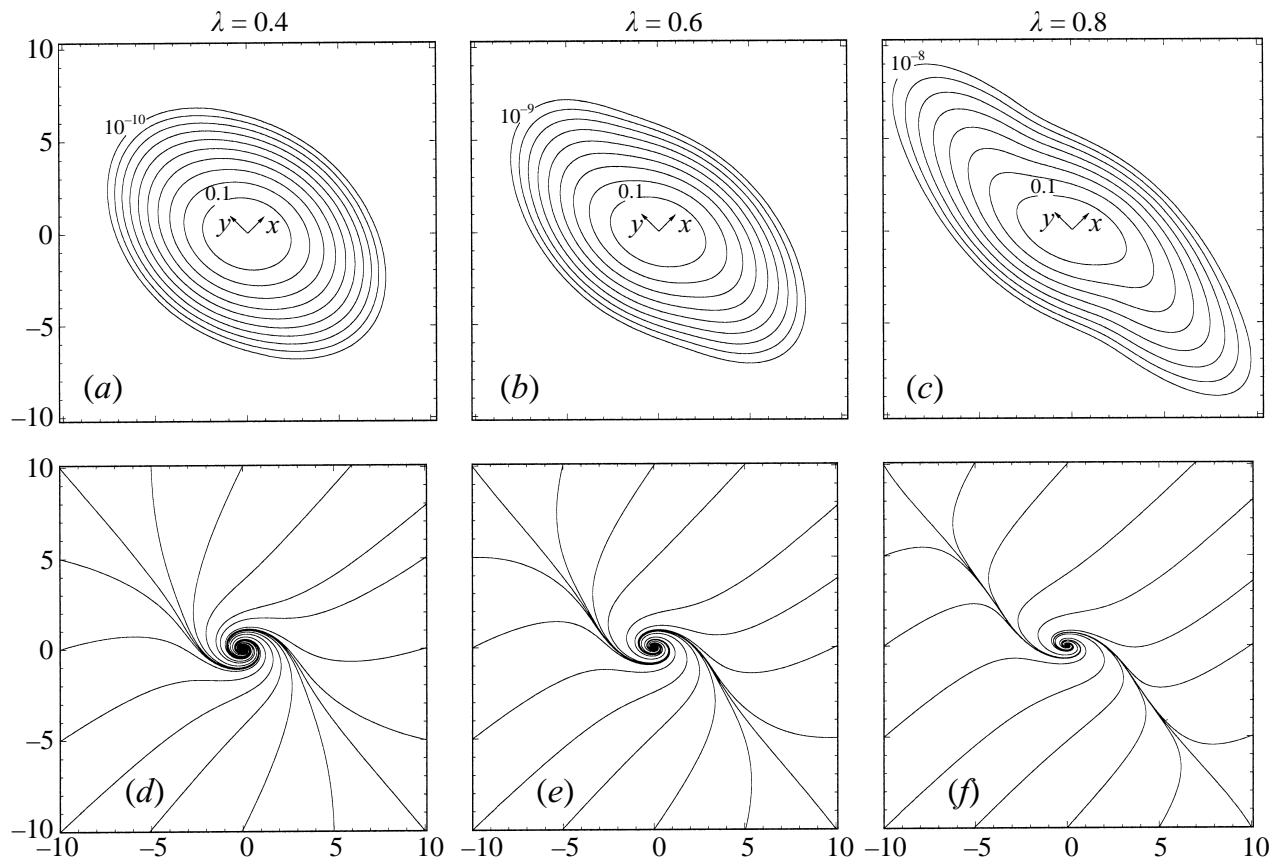

FIGURE 1. Isovorticity contours $(a-c)$ and projected streamlines $(d-f)$ at $R=10, \lambda=0.4,0.6,0.8$

Note rotation of $(x, y)$ axes.

For each case studied, tables $2-5$ show the value of the core vorticity and the largest value of the vorticity at the domain boundary. Ideally, this latter quantity should be at the level of machine precision. In figures $1-4$, the isovorticity contours and the streamlines projected onto the plane normal to $\omega$ are plotted on the domain on which they are calculated. The isovorticity contour plots show the directions of the principal axes of strain and contours varying from $0.1 R$ to the lowest value, given separately on each plot. The projected streamlines correspond to globally inward flow when $\lambda \leqslant 1$ but for $\lambda>1$ some of the contours shown correspond to fluid particles that are not being swept into the vortex. Our results can be classified into three categories, denoted by low, intermediate, or high Reynolds numbers.

In the low-Reynolds-number region, $R=1-10$ (table 2, figure 1) the axes of strain are rotated by $45^{\circ}$ to achieve maximum accuracy. All plots are shown on the domain of calculation and the principal axes of strain are shown at the origin of each isovorticity plot. The vorticity contours are stretched in the $y$-direction and so steady solutions cannot be accurately calculated within our bounded domain beyond $\lambda>0.8$. This behaviour can be predicted by assuming that low-Reynolds-number flow 


\begin{tabular}{cccccc}
\hline$\lambda$ & $M$ & $N$ & $\phi$ & $\omega(r=0)$ & $\left.\omega_{\max }\right|_{\text {boundary }}$ \\
0.5 & 40 & 40 & 0 & 0.9993 & $1.79 \times 10^{-12}$ \\
1.0 & 50 & 50 & 0 & 0.9974 & $6.19 \times 10^{-15}$ \\
1.5 & 40 & 40 & $\pi / 3$ & 0.9939 & $1.08 \times 10^{-8}$ \\
2.0 & 50 & 50 & $-\pi / 6$ & 0.9888 & $4.95 \times 10^{-6}$ \\
2.5 & 20 & 80 & 0 & 0.9811 & $9.13 \times 10^{-5}$
\end{tabular}

TABLE 3 . Vorticity values at the core and the boundary for $R=100$.
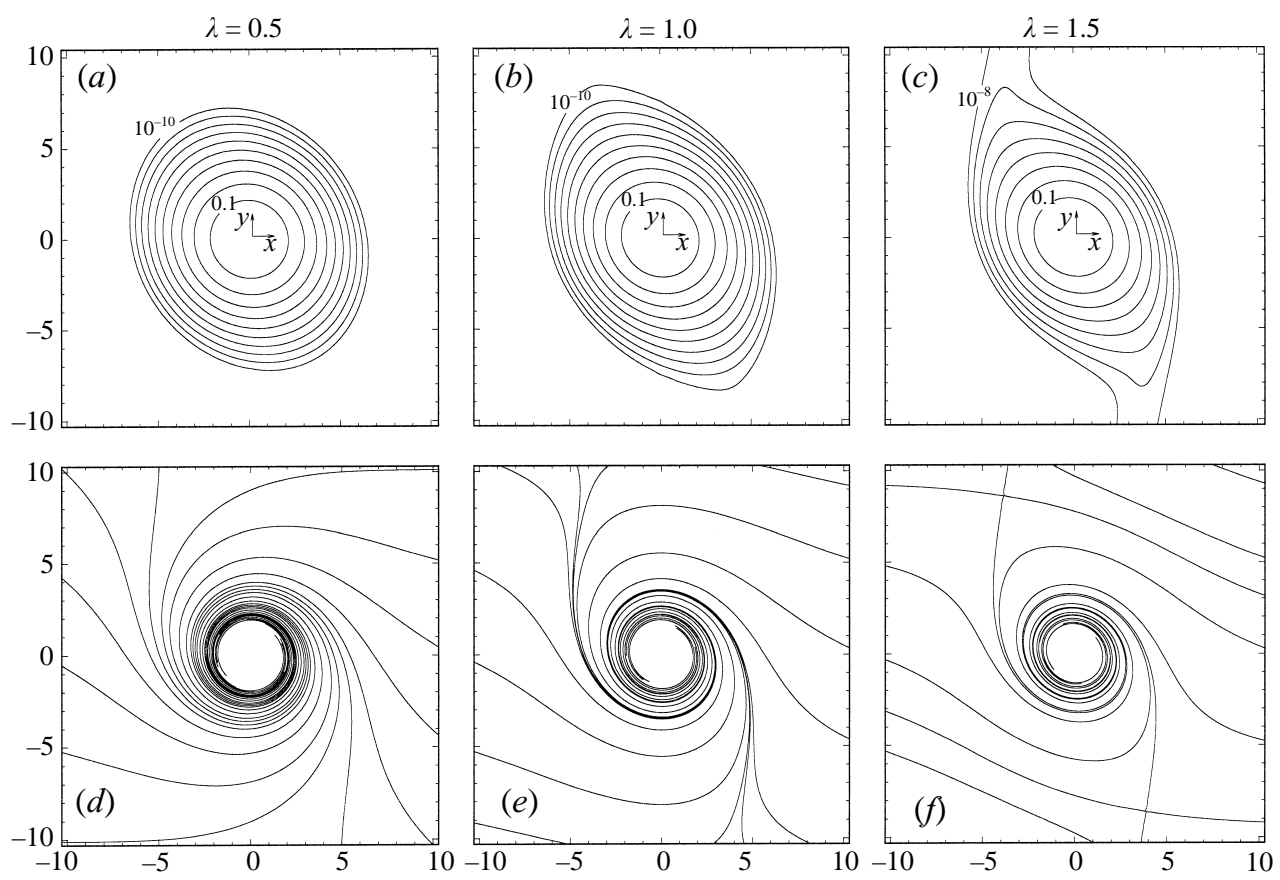

FIGURE 2. Isovorticity contours $(a-c)$ and projected streamlines $(d-f)$ at $R=100, \lambda=0.5,1.0,1.5$.

can be approximated by the zero-Reynolds-number limit (2.4) and it is likely that this is why RS84 stated that as $\lambda \rightarrow 1$ the 'elongation of the vortex tends to infinity' and only investigated solutions for $\lambda \leqslant 0.75$. The streamlines are shown for completeness and for comparison to higher-Reynolds-number cases. See Bajer \& Moffatt (1997) for similar streamline patterns in the context of a stretched magnetic-flux tube.

For intermediate values of the Reynolds number, $R=100$ (table 3, figure 2), it is clear that elongation of the vortex does not tend to infinity as $\lambda \rightarrow 1$. In fact, increasing the circulation tends to reduce the ellipticity of the vortex as well as rotating the major and minor axes of the vortex at an angle that approaches $45^{\circ}$ for large $R$ (originally noted in RS84). This enables a numerically steady solution to be found for $\lambda=1$, as shown in figure 2(b), which, in contrast to the vortex sheet (1.5), is a bounded vorticity solution in plane strain. Furthermore, at this Reynolds number, we are easily able to find numerically steady solutions for $\lambda>1$. The solution for $\lambda=1.5$ is shown in figure 2(c), but extra care must be taken for solutions in this region. As shown in figure $2(f)$, for $\lambda>1$, stagnation points will appear in the flow. MKO94 argued that the vorticity beyond the stagnation points will be transcendentally small 


$\begin{array}{ccc}\lambda & \omega(r=0) & \left.\omega_{\max }\right|_{\text {boundary }} \\ 1.0 & 0.99997 & 1.96 \times 10^{-14} \\ 2.0 & 0.99989 & 2.01 \times 10^{-14} \\ 3.0 & 0.99976 & 2.44 \times 10^{-14} \\ 6.0 & 0.99905 & 1.26 \times 10^{-14}\end{array}$

TABLE 4 . Vorticity values at the core and the boundary $R=1000$ with $M, N=50, \phi=0$.
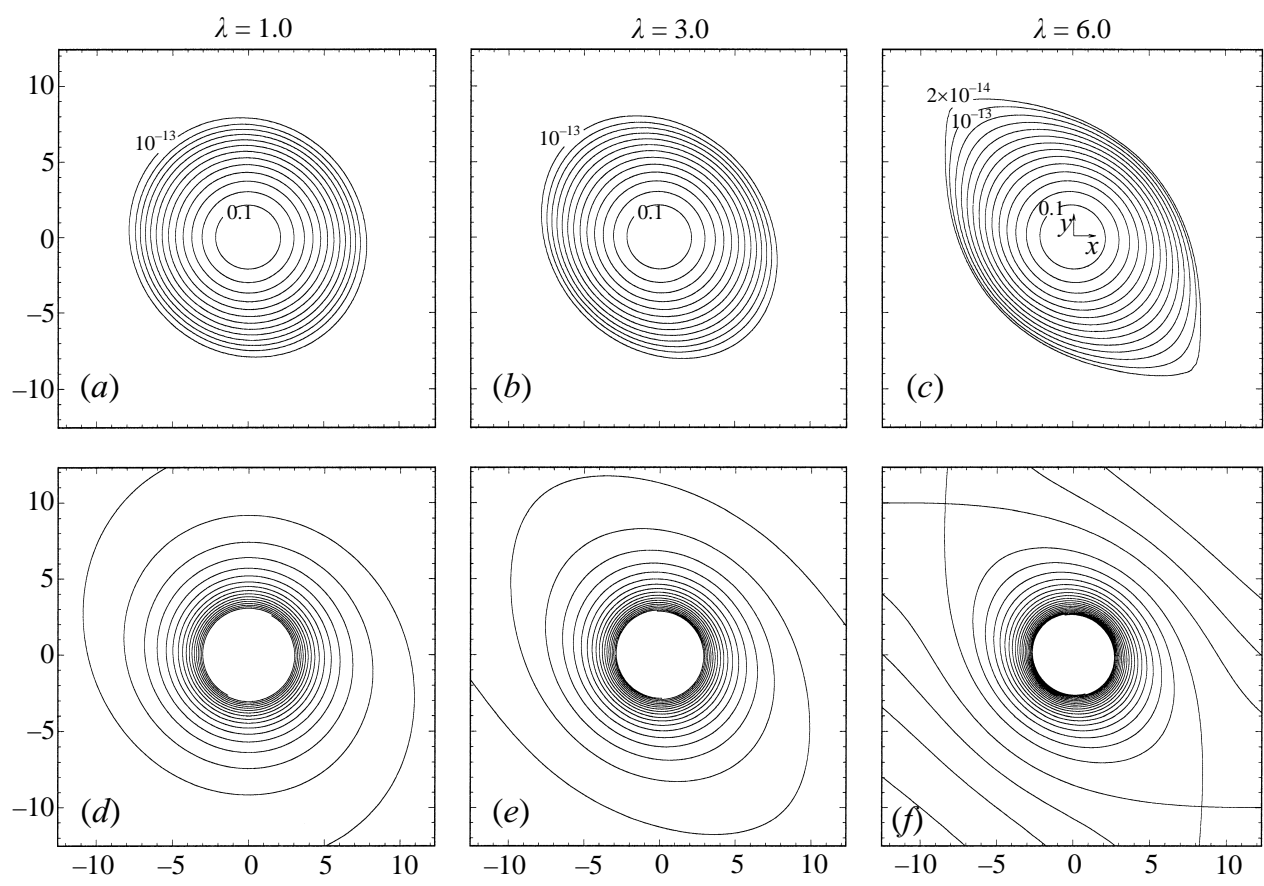

FiguRE 3. Isovorticity contours $(a-c)$ and projected streamlines $(d-f)$ at $R=1000, \lambda=1,3,6$.

and therefore be convected away from the vortex as a passive scalar. However, their estimate for vorticity flux due to this phenomenon is exceedingly small and this time variation does not seem to compromise our ability to find numerically steady solutions for $\lambda>1$. Instead, our solutions correspond to a 'snapshot' of the vorticity at a time when the majority of the vorticity is contained within our bounded domain. To account for this, we will call our steady solutions 'quasi-steady' and later, in $\S 3$, address the time variation and the nature of the vorticity flux.

For very large Reynolds numbers, $R=10^{3}-10^{4}$ (tables $4-5$, figures 3 and 4), variation away from the the Burgers solution (2.3) is very small except for large strain ratios, well into the biaxial region. In this region, we notice that the vorticity contours closely relate to the projected streamlines (see figure 4). Since the vorticity is exponentially small outside the core, it is being passively convected by the velocity field. The projected streamlines closely resemble the streamlines for a point vortex in pure two-dimensional strain, except that since the vortex is being stretched, there is some inward flow which causes the streamlines to slowly coil into the origin. 


\begin{tabular}{rll}
\multicolumn{1}{c}{$\lambda$} & $\omega(r=0)$ & $\left.\omega_{\max }\right|_{\text {boundary }}$ \\
50.0 & 0.99934 & $2.44 \times 10^{-14}$ \\
100.0 & 0.99734 & $1.89 \times 10^{-8}$ \\
150.0 & 0.9940 & $1.26 \times 10^{-4}$
\end{tabular}

TABLE 5. Vorticity values at the core and the boundary $R=10^{4}$ with $M, N=50, \phi=0$.
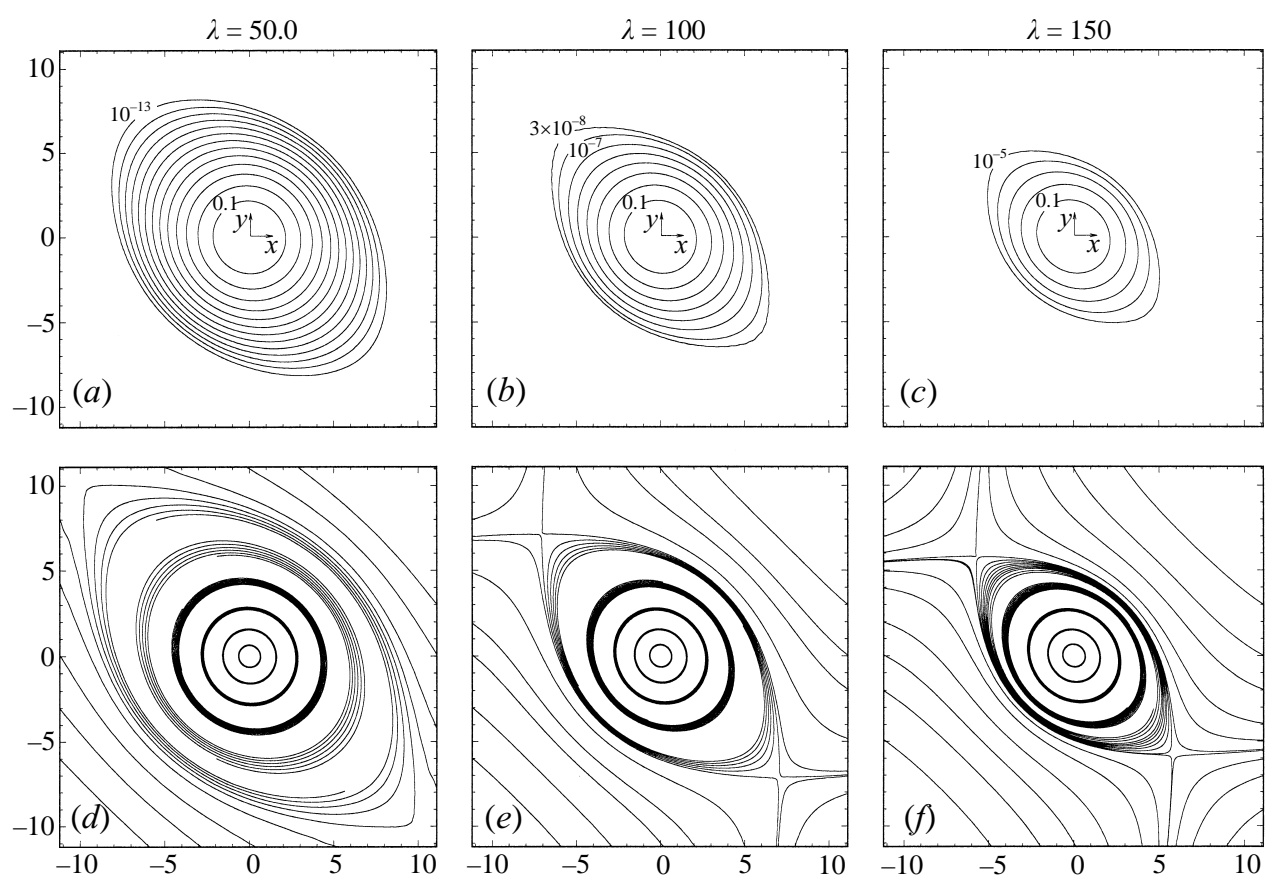

FIGURE 4. Isovorticity contours $(a-c)$ and projected streamlines $(d-f)$ at $R=10^{4}, \lambda=50,100,150$.

\section{Biaxial strain at large Reynolds number}

\subsection{Characteristic regions of the flow}

We observe from our numerical results that for $R \gg 1$ the vortex becomes increasingly circular which makes the axisymmetric Burgers vortex a very good approximation to the flow until the strain ratio becomes large enough such that outside the core of the vortex, the isovorticity contours resemble figure 5. This figure shows several key characteristic regions of the flow. Region I, the core of the vortex, within $r=O$ (1), is where viscous diffusion of vorticity is balanced by the intensification of vorticity by vortex stretching, and the streamlines and isovorticity contours are nearly circular. Region II, which we will call the cat's-eye, due to its shape, is defined as the interior region between the stagnation points of the flow, $1<r<\varepsilon^{-1 / 2}$, when the strain ratio is large, $\lambda \gg 1$, the Reynolds number is large, $R \gg 1$, but their ratio is small,

$$
\varepsilon \equiv \frac{\lambda}{R} \ll 1 \text {. }
$$

It was noted earlier that for $\lambda>3$, the vortex, when rotated in $(x, y, z)$ space away from the $z$-axis, is subject to a tilt instability on a time scale of $O\left((\beta-\gamma)^{-1}\right)$. This 


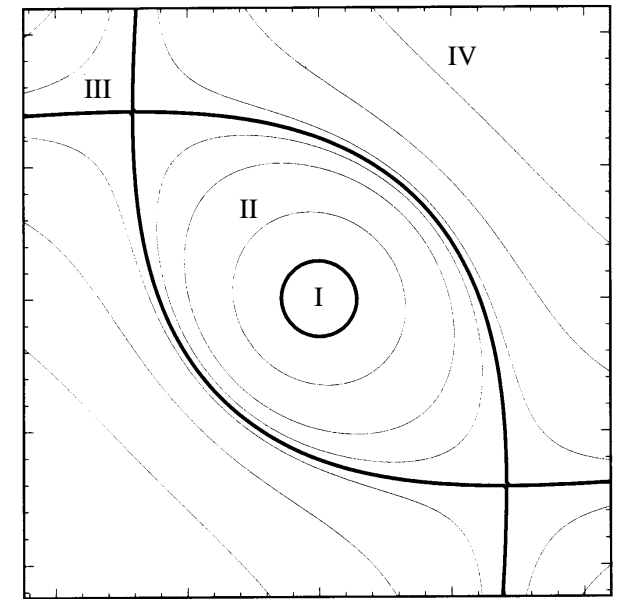

FIGURE 5. Schematic of the two-dimensional asymptotic limit of projected streamlines that divide the characteristic regions of the nearly two-dimensional flow for $\lambda \gg 1, R \gg 1$ and $\varepsilon \ll 1$.

time scale is of the same order as that associated with strain-vortex-induced motions in the $(x, y)$-plane at a radius $r \sim O\left(\varepsilon^{-1 / 2}\right)$, the boundary of region II. The present asymptotic results, which assume precise alignment of the vortex axis with the $z$ axis, are nonetheless of interest because first a vorticity distribution of unusual and unexpected form arises in region II, secondly we find a time scale for decay of the vortex when $\lambda>1$ significantly different to MKO94, and third, our results are relevant to certain unsteady but strictly two-dimensional flows which can obtained from the stretched vortex flow using the Lundgren transformation (Lundgren 1982).

In region II, the vorticity is exponentially small and is basically convected along streamlines. The flow in the $(x, y)$-plane is almost two-dimensional. The cat's-eye shape will accurately describe the contours of the vorticity and almost describe the streamlines, as shown, for example, in figures $3(c, f)$ and 4 . The velocity in this region is dominated by the core vorticity and the external strain. Region III begins at each stagnation point and extends away from the vortex. Vorticity in this region is also exponentially small, but it is convected away by the strain, which begins to dominate the induced velocities from the core. Region IV is the far field where structure of the vortex becomes unimportant.

We develop an asymptotic form for the vorticity in Region II and use this to determine the rate of decrease in circulation out of the cat's-eye. Then, we consider the nature of the vorticity leakage through Region III and match the rate of circulation increase in Region IV to the flux of vorticity from the cat's-eye.

\subsection{Region II, the cat's-eye}

\subsubsection{Vorticity distribution}

We can write the non-dimensional vorticity equation (2.1) in its steady form in two parts by considering the strain to consist of the sum of an axially symmetric three-dimensional part and a two-dimensional pure strain part,

$$
L_{0}(\omega)+L_{1}(\omega)=0,
$$


where

$$
\begin{aligned}
& L_{0}(\omega)=\frac{\partial^{2} \omega}{\partial x^{2}}+\frac{\partial^{2} \omega}{\partial y^{2}}+x \frac{\partial \omega}{\partial x}+y \frac{\partial \omega}{\partial y}+2 \omega, \\
& L_{1}(\omega)=(\lambda x-R u) \frac{\partial \omega}{\partial x}+(-\lambda y-R v) \frac{\partial \omega}{\partial y} .
\end{aligned}
$$

The terms of $L_{0}$ represent the balance of viscous diffusion of vorticity and intensification of vorticity by vortex stretching in an axisymmetric strain while the terms of $L_{1}$ correspond to the effective two-dimensional transport of vorticity by the induced velocity and by the two-dimensional, or in-plane, strain. Focusing first on $L_{1}$, we hypothesize that the velocity field given by the bracketed terms in (3.4) in the region outside the core of the vortex, $r>1$, can be represented to leading order by the potential flow of a point vortex in the pure two-dimensional strain. The streamfunction and velocity for this potential flow can be written as

$$
\psi_{0}(x, y)=-\ln \left(x^{2}+y^{2}\right)^{1 / 2}-\varepsilon x y .
$$

The stagnation points of this flow occur where the velocities induced by the point vortex and the strain balance,

$$
\begin{aligned}
& x_{s}= \pm(1 / 2 \varepsilon)^{1 / 2}, \\
& y_{s}=\mp(1 / 2 \varepsilon)^{1 / 2},
\end{aligned}
$$

or, in polar coordinates,

$$
r_{s}=\varepsilon^{-1 / 2}, \quad \theta=\frac{3 \pi}{4},-\frac{\pi}{4} .
$$

The true projected streamlines for the numerical solutions of $\S 2$ show a nearly cat's-eye form where $\lambda$ and $R$ are given by (3.1), as can be seen in figure 4 .

We remark that for the full three-dimensional straining field, the $(x, y)$-plane velocities will balance at

$$
\begin{aligned}
& x_{s}= \pm\left(\frac{\lambda-1}{\lambda+1}\right)^{1 / 4}(1 / 2 \varepsilon)^{1 / 2}, \\
& y_{s}=\mp\left(\frac{\lambda+1}{\lambda-1}\right)^{1 / 4}(1 / 2 \varepsilon)^{1 / 2},
\end{aligned}
$$

or, in polar coordinates,

$$
\begin{aligned}
& r_{s}=\left(\frac{\lambda^{2}}{\lambda^{2}-1}\right)^{1 / 4} \varepsilon^{-1 / 2}, \\
& \theta=\frac{3 \pi}{4}-\frac{1}{2 \lambda}+O\left(\lambda^{-3}\right), \quad-\frac{\pi}{4}-\frac{1}{2 \lambda}+O\left(\lambda^{-3}\right),
\end{aligned}
$$

so that comparison with (3.8) will require that $\lambda$ be large.

Next we put

$$
\begin{aligned}
\Psi & =\psi_{0}+\psi_{1}, \\
\psi_{1} & =\ln \left(x^{2}+y^{2}\right)^{1 / 2}+\psi,
\end{aligned}
$$

where $\psi_{1}$ is a perturbation streamfunction associated with the exponentially small vorticity $\omega=\omega_{I I}$ outside the core and $\psi$ is the dimensionless form of the streamfunction in (2.2). From (2.2) $\psi_{1}$ satisfies

$$
\nabla^{2} \psi_{1}=-\omega .
$$


The streamfunction $\Psi$ gives the whole of the two-dimensional flow corresponding to the velocity components of (3.4) so that

$$
-\lambda x+R u=R \frac{\partial \Psi}{\partial y}, \quad \lambda y+R v=-R \frac{\partial \Psi}{\partial x} .
$$

When (3.13) and (3.16) are used in (3.4), the vorticity equation in Region II may be written as

$$
\begin{gathered}
L_{0}(\omega)+L_{2}(\omega)+L_{3}(\omega)=0, \\
L_{2}(\omega)=-R\left(\frac{\partial \psi_{0}}{\partial y} \frac{\partial \omega}{\partial x}-\frac{\partial \psi_{0}}{\partial x} \frac{\partial \omega}{\partial y}\right), \\
L_{3}(\omega)=-R\left(\frac{\partial \psi_{1}}{\partial y} \frac{\partial \omega}{\partial x}-\frac{\partial \psi_{1}}{\partial x} \frac{\partial \omega}{\partial y}\right) .
\end{gathered}
$$

For large $R$, when $1<r<\varepsilon^{-1 / 2}$, we argue that both $\omega$ and $\psi_{1}$ will be exponentially small, with the consequence that the dominant terms of the equation will be $L_{2}(\omega)$. Solving $L_{2}(\omega)=0$ will require that

$$
\omega=\omega_{I I}\left(\psi_{0}\right)=\omega\left(-\ln \left(x^{2}+y^{2}\right)^{1 / 2}-\varepsilon x y\right) .
$$

We must now determine the function of $\psi_{0}$ in (3.20) that satisfies (3.3) and (3.18) to leading order. We develop a heuristic argument for an expansion of appropriate form. First we assume that, owing to what will be shown to be exponentially small vorticity in Region II, the terms of $L_{0}$ and $L_{2}$ will be dominant over $L_{3}$ in this region. The self-consistency of this assumption is demonstrated in Appendix A. Next we seek a form for $\omega_{I I}$ which will approach the Burgers limit $\omega_{B V}=\exp \left(-r^{2} / 2\right)$ when $\varepsilon \rightarrow 0$. In this limit, with $r \gg 1$ and $\psi_{B V} \equiv \lim _{\varepsilon \rightarrow 0}\left(\psi_{0}\right)$, eliminating $r$ from (3.5) and $\omega_{B V}$ gives

$$
\omega_{B V}=\exp \left[-\frac{1}{2} \exp \left(-2 \psi_{B V}\right)\right] \text {. }
$$

When $\varepsilon \rightarrow 0$ we require that $\omega_{I I}\left(\psi_{0}\right)$ in (3.20) agree with (3.21). This requirement immediately suggests that in region II, when $0<\varepsilon \ll 1$, (3.20) takes the form

$$
\omega_{I I}\left(\psi_{0}\right)=\exp \left[-\frac{1}{2} \exp \left(-2 \psi_{0}\right)\right]=\mathrm{e}^{-r^{2} \exp \left(\varepsilon r^{2} \sin 2 \theta\right) / 2}+\cdots,
$$

where a conversion to polar coordinates has been used. Equation (3.22) in turn motivates an expansion of the form

$$
\omega_{I I}(r, \theta)=\mathrm{e}^{-r^{2} \exp \left(\varepsilon r^{2} \sin 2 \theta\right) / 2}\left(g_{1}(r)+\varepsilon g_{2}(r) \sin 2 \theta \ldots\right),
$$

and we note that (3.23) has the form suggestive of an expansion beyond all orders (see Berry 1991). This is made clearer if we introduce a transformation of the form $\tilde{r}=\varepsilon^{1 / 2} r$, but since this offers no advantage at leading order within the bracketed factor in (3.23), we do not follow this path. It is clear from the method of construction that (3.23) satisfies (3.18) to $O\left(\varepsilon^{0}\right)$ within the bracketed factor, provided $g_{1}=1$.

We must now demonstrate that (3.3) is also satisfied to leading order, with $g_{1}(r)=1$. In polar coordinates, this equation becomes

$$
\frac{\partial^{2} \omega}{\partial r^{2}}+\frac{1}{r} \frac{\partial \omega}{\partial r}+\frac{1}{r^{2}} \frac{\partial^{2} \omega}{\partial \theta^{2}}+r \frac{\partial \omega}{\partial r}+2 \omega=0 .
$$

When (3.23) is substituted into (3.24), the $\mathrm{e}^{-r^{2} \exp \left(\varepsilon r^{2} \sin 2 \theta\right) / 2}$ factor cancels uniformly, 
and expanding the resulting equation in powers of $\varepsilon$, we find to $O\left(\varepsilon^{0}\right)$,

$$
\left(\frac{1}{r}-r\right) \frac{\partial g_{1}}{\partial r}+\frac{\partial^{2} g_{1}}{\partial r^{2}}=0
$$

The general solution of (3.25) may be written as a constant plus a second constant times an exponential integral. This second term may be shown to be unbounded when $r \rightarrow 0$ and $r \rightarrow \infty$. Hence the only finite solution is $g_{1}=$ const $=1$ as required. Thus, to leading order (3.3) and (3.18) are satisfied by (3.22). We remark that (3.24) can be satisfied to $O\left(\varepsilon^{0}\right)$ in the above sense by a more general form of (3.23) with $g_{1}(r)=1$ and the exponential prefactor replaced by the form $\mathrm{e}^{-r^{2} \exp (\varepsilon Q(r, \theta)) / 2}$ for a wide class of functions $Q(r, \theta)$. Compatibility with (3.20) is however a severe restriction, and a unique solution appears to be $Q(r, \theta)=r^{2} \sin 2 \theta$.

The difficult task of determining the next term at $O(\varepsilon)$ in the bracketed factor in (3.23) is not attempted here. We suspect that the dominant balance arguments used at the leading order do not apply at higher order and that the relevant terms of $L_{0}, L_{1}$ and $L_{3}$ must be combined. Our leading-order solution is formally valid only in $1<r<\varepsilon^{-1 / 2}$. However comparison with the present numerical solutions, to be discussed subsequently, show that (3.22) is an extremely good approximation right up to the cat's eye boundary $r=O\left(\varepsilon^{-1 / 2}\right)$, thus providing strong evidence that neglected higher-order terms do not lead to disordering in Region II.

Expansion of (3.22) in orders of $\varepsilon$ gives

$$
\omega_{\mathrm{II}}(r, \theta)=\mathrm{e}^{-r^{2} / 2}\left[1-(\varepsilon \sin 2 \theta) \frac{r^{4}}{2}+O\left(\varepsilon^{2}\right)\right],
$$

which matches, when $r \rightarrow 0$, to the MKO94 asymptotic solution for $R \gg 1$ (in terms of their scaling definitions)

$$
\begin{gathered}
\omega=\omega_{0}+\varepsilon \Omega(\hat{r}) \sin 2 \theta+O\left(\varepsilon^{2}\right), \\
\Omega(\hat{r}) \sim \frac{1}{16} \hat{r}^{4} \mathrm{e}^{-r^{2} / 4}
\end{gathered}
$$

as $r \rightarrow \infty$. In order to remedy the disordering of terms of the MKO94 solution at $r=(2 / \varepsilon)^{1 / 4}$, Jiménez et al. (1996) performed an asymptotic analysis involving the Lundgren transformation and coordinate stretching that delayed the disordering to $r=\varepsilon^{-1 / 2}$ (again in terms of their definitions, which involve time dependence due to the Lundgren transformation),

$$
\omega(\hat{r}, \theta)=\frac{1}{4 \pi t} \mathrm{e}^{-\left(\hat{r}-\varepsilon \hat{r}^{3} \pi \sin 2 \theta\right)^{2} / 4 t}+O\left(\varepsilon^{2}\right) .
$$

Again, (3.22) matches their form to $O\left(\varepsilon^{2}\right)$. Figure $6(a, b)$ shows that the agreement between (3.22) and numerics for $R=1000, \lambda=50,100$ is good. The vorticity at the stagnation point (and at any point along the cat's-eye boundary) given by (3.22) is $\omega_{s}=\exp (-1 / 2 e \varepsilon)$. This value is compared to numerics in table 6 for cases in which the stagnation point appears within the domain of validity of the quasi-steady calculation. The stagnation point is used because it appears at the limit of the region of the validity of (3.22), yet the agreement is still very good.

A comparison of (3.22) with the asymptotic formulations from MKO94 (3.27), Jiménez et al. (1996) (3.29), and the present numerical solutions along cuts $\theta=\pi / 4$ 


\begin{tabular}{ccccc}
\hline$R$ & $\lambda$ & $\omega_{I I}$ & $\omega_{\text {numerics }}$ \\
$10^{3}$ & 6.0 & $1.0210^{-14}$ & $4.8510^{-14}$ \\
$10^{4}$ & 100 & $9.8910^{-9}$ & $1.0310^{-8}$ \\
$10^{4}$ & 150 & $1.2910^{-6}$ & $4.7210^{-6}$ \\
\multicolumn{5}{c}{ TABLE } \\
6. Stagnation point vorticity \\
\hline
\end{tabular}
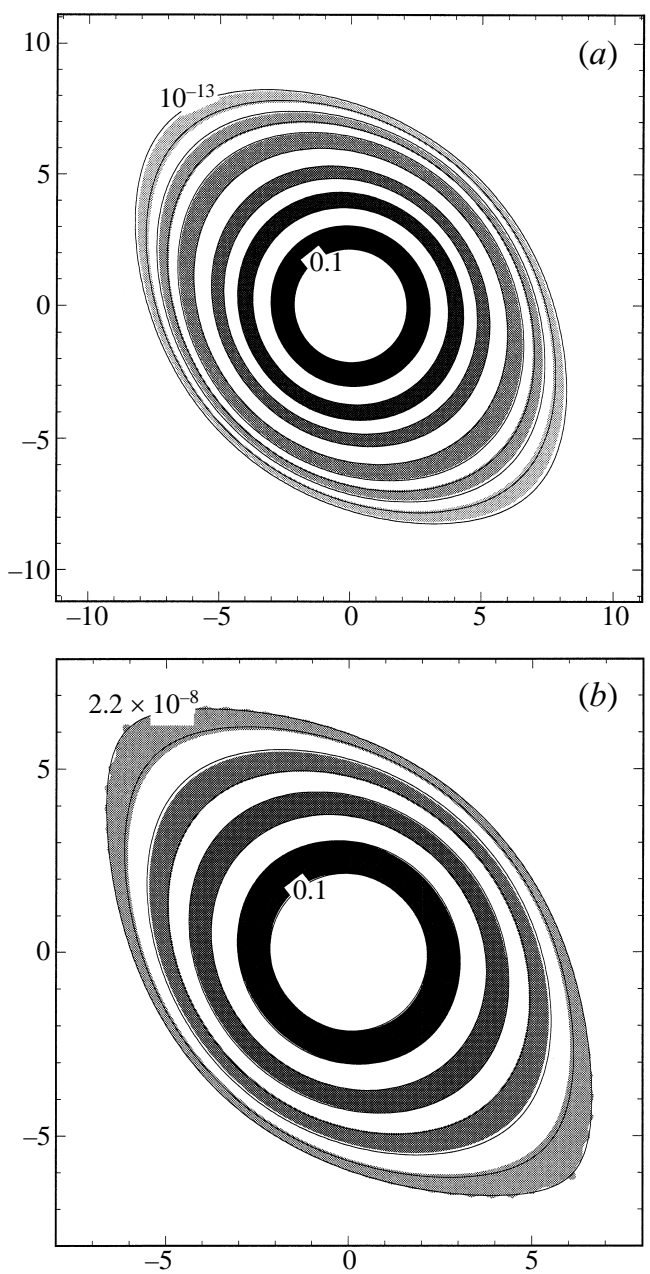

FIGURE 6. Comparison of isovorticity contours of numerical results (shaded regions) to equation (3.22) (solid lines) for $R=10^{4}$, and (a) $\lambda=50$, (b) $\lambda=100$.

and $\theta=-\pi / 4$ are shown in figure $7(a)$ and $7(b)$ respectively. Along $\theta=\pi / 4$, all theories give good agreement to the numerics except MKO94 which changes sign at $r \approx 3.76$. Along $\theta=-\pi / 4$, figure $7(b)$ clearly shows that (3.22) is the only form that is accurate to the edge of the cat's eye.

In summary, in Region II the essential properties of the flow are that the core of the vortex can be closely approximated by a point vortex and that the exponentially small 

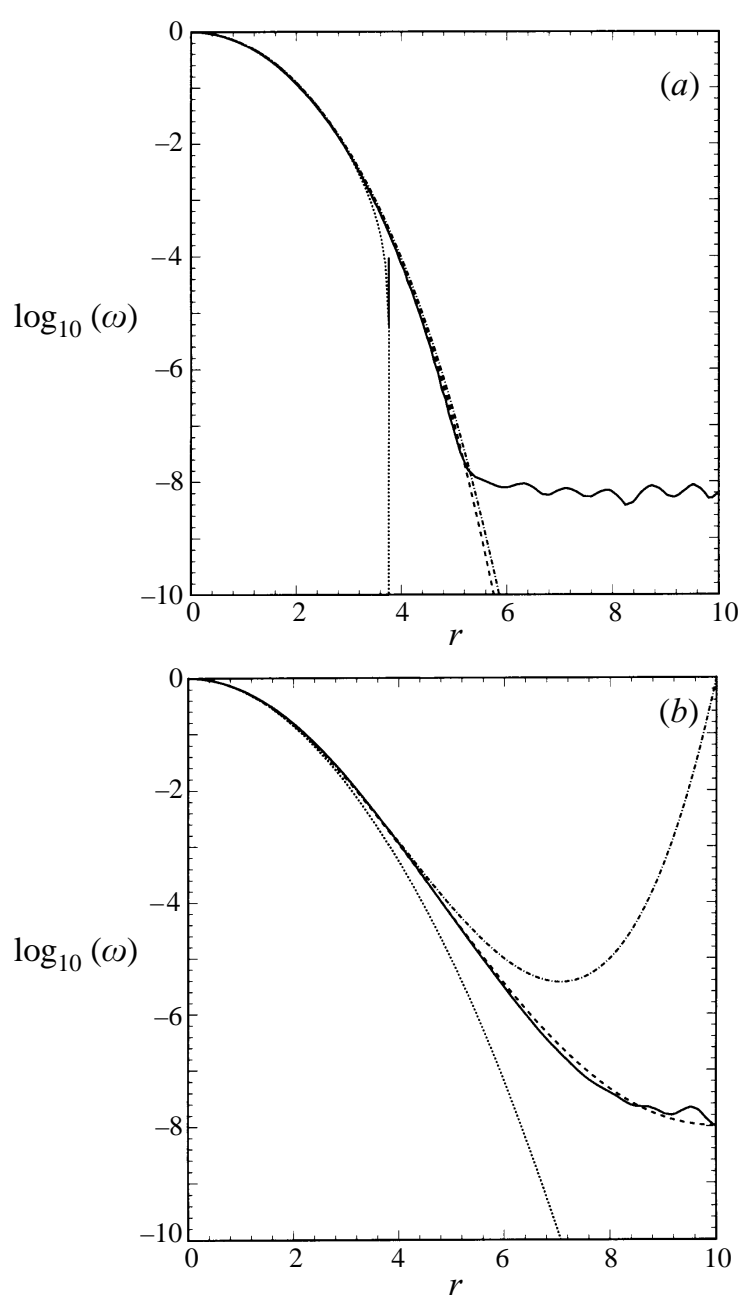

FIGURE 7. Vorticity along (a) $\theta=\pi / 4$ and $(b) \theta=-\pi / 4$ from MKO94 (3.27) (dotted), Jiménez et al. (1996) (3.29) (dash-dot), our result (3.22) (dashed), and numerics (solid) for $\lambda=100, R=10,000$, $\varepsilon=0.01$. In $(a)$ the MKO94 result becomes negative at $r=3.76$.

vorticity is being convected by the background strain, which is composed of stretching and $(x, y)$-plane components. Our main result is (3.22). This $\omega_{I I}$ satisfies both (3.3) and (3.18) to leading order, and matches the inner expansions of both MKO94 and Jiménez et al. in region I. Moreover (3.22) agrees well with our numerical results uniformly in Region II, providing strong evidence that neglected higher-order terms do not lead to disordering on the cat's eye boundary.

\subsubsection{A generalization}

Our approach is not limited to the case of biaxial strain. For example, given the Burgers vortex embedded in a strain field in which $\psi_{0}$ of (3.5) is replaced by $\psi_{0}^{(n)}(r, \theta)=-\ln (r)-\varepsilon_{n} r^{n} \sin n \theta, n>1$, where $\varepsilon_{n}$ is a measure of the relative strength of the strain field, the asymptotic form of the vorticity outside of the vortex core but 
(a)

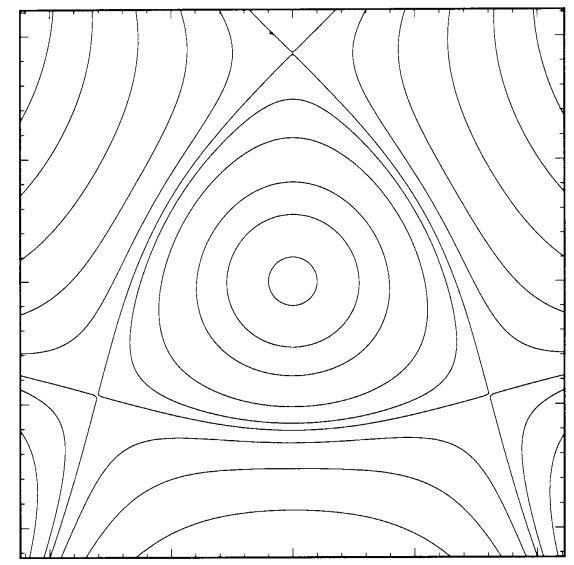

(b)

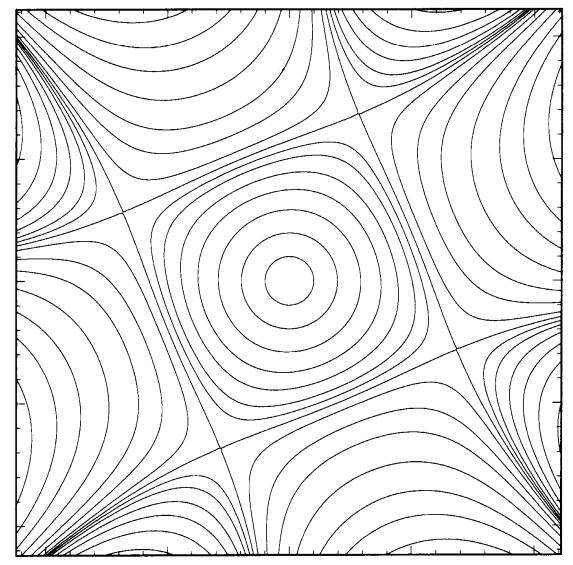

FIGURE 8. Asymptotic limit of the Burgers vortex in a straining field where (a) $\psi_{0}^{(3)}(r, \theta)=-\ln (r)-\varepsilon_{3} r^{3} \sin 3 \theta$ and $(b) \psi_{0}^{(4)}(r, \theta)=-\ln (r)-\varepsilon_{4} r^{4} \sin 4 \theta$.

within some dividing streamline will be,

$$
\begin{gathered}
\omega_{I I}=\exp \left[-\frac{1}{2} r^{2} \exp \left(-2 \varepsilon_{n} r^{n} \sin n \theta\right)\right], \\
\Psi=-\ln (r)-\varepsilon_{n} r^{n} \sin n \theta+\psi_{1}+O\left(\varepsilon_{n}^{2}\right), \\
\nabla^{2} \psi_{1}=-\omega_{I I} .
\end{gathered}
$$

The cat's-eye shape corresponds to $n=2$, and two other examples for $n=3$ and $n=4$ are shown in figures $8(a)$ and $8(b)$ where the asymptotic form of the vortex exhibits triangular and square dividing streamlines, respectively. Clearly, for $\psi_{n}$, the dividing streamline corresponds to an $n$-sided polygon at a radius of $\varepsilon_{n}^{-1 / n}$ from the origin. It should be noted that for each case the asymptotic form of the vortex core (Region I) could be calculated in a manner analogous to MKO94.

\subsection{Slow decay of vortex for $\lambda \gg 1$}

Although the solutions we find are nearly steady, in fact numerically steady, there is extremely slow time variation in the form of flux of vorticity to infinity. This slow time variation was originally noted in MKO94 where they hypothesized that, in biaxial strain, diffusion would not be able to balance the strain. We perform a circulation decay rate estimate by considering flux of vorticity across the cat's-eye boundary and then analyse the structure of vorticity leaking into Region IV.

\subsubsection{Flux of vorticity out of the cat's-eye}

By integrating the dimensionless vorticity equation (2.1) over an area, $A$, and using Green's theorem to relate area integrals to contour integrals, the time derivative of the circulation is easily found to be the sum of diffusion of vorticity and transport of vorticity across the boundary of the domain,

$$
\frac{\partial \Gamma}{\partial t} \equiv \frac{\partial}{\partial t} \iint_{A} \omega \mathrm{d} A=\oint_{\partial A}(\nabla \omega \cdot \boldsymbol{n}) \mathrm{d} l-\oint_{\partial A}(\boldsymbol{u} \cdot \boldsymbol{n}) \omega \mathrm{d} l,
$$

where $\boldsymbol{n}$ is an outward-facing unit vector normal to the boundary and $\boldsymbol{u}$ is the velocity at the boundary. It will be useful to write the velocity decomposed into a 
two-dimensional part induced by vorticity and pure two-dimensional strain, and a part associated with stretching,

$$
\boldsymbol{u}=\boldsymbol{u}_{1}+\boldsymbol{u}_{2}=\left(\begin{array}{c}
+\partial \psi / \partial y \\
-\partial \psi / \partial x
\end{array}\right)+\left(\begin{array}{c}
-x \\
-y
\end{array}\right) .
$$

If we choose the boundary $A$ to be the edge of the cat's-eye, i.e. the contour defined by

$$
\ln \left(\varepsilon r^{2}\right)+\varepsilon r^{2} \sin 2 \theta=-1,
$$

the dominant velocities will only come from $\psi \sim \psi_{0}(x, y)=-\ln \left(x^{2}+y^{2}\right)^{1 / 2}-\varepsilon x y$, so that

$$
\boldsymbol{u}=\boldsymbol{u}_{1}+\boldsymbol{u}_{2}=\left(\begin{array}{l}
+\partial \psi_{0} / \partial y \\
-\partial \psi_{0} / \partial x
\end{array}\right)+\left(\begin{array}{l}
-x \\
-y
\end{array}\right) .
$$

Along the cat's-eye, the unit normal vector is defined to be

$$
\boldsymbol{n}=\frac{\nabla \psi_{0}}{\left(\nabla \psi_{0} \cdot \nabla \psi_{0}\right)^{1 / 2}}=-\frac{\nabla \omega_{I I}}{\left(\nabla \omega_{I I} \cdot \nabla \omega_{I I}\right)^{1 / 2}}
$$

while

$$
\begin{aligned}
\boldsymbol{u} \cdot \boldsymbol{n} & =\left(\frac{\partial \psi_{0}}{\partial y} \frac{\partial \psi_{0}}{\partial x}-\frac{\partial \psi_{0}}{\partial x} \frac{\partial \psi_{0}}{\partial y}\right)+\boldsymbol{u}_{2} \cdot \boldsymbol{n} \\
& =\boldsymbol{u}_{2} \cdot \boldsymbol{n} .
\end{aligned}
$$

Also, along this contour the cat's-eye vorticity from (3.22) is a constant, $\omega_{c}=$ $\exp (-1 / 2 e \varepsilon)$, so that we have

$$
\frac{\partial \Gamma}{\partial t}=\omega_{c} \oint_{\partial A}\left(\frac{\nabla \omega_{I I}}{\omega_{c}}-\boldsymbol{u}_{2}\right) \cdot \boldsymbol{n} \mathrm{d} l .
$$

Upon putting $\rho=\varepsilon^{1 / 2} r$, and solving (3.35) for $\theta(\rho)$, we find that the circulation decrease in terms of dimensional variables is

$$
\frac{\partial \Gamma}{\partial t}=-\frac{c}{4 \pi} \gamma \varepsilon^{-1} \Gamma \exp \left(\frac{-1}{2 e \varepsilon}\right)
$$

where $c$ is a pure number defined by the integral along the cat's eye, in terms of $\rho$,

$$
c=4 \int_{m}^{1}\left(\frac{\nabla \omega_{I I}(\rho)}{\omega_{c}}-\boldsymbol{u}_{2}(\rho)\right) \cdot \boldsymbol{n}(\rho)\left[1+\rho^{2}\left(\frac{\mathrm{d} \theta}{\mathrm{d} \rho}\right)^{2}\right]^{1 / 2} \mathrm{~d} \rho,
$$

and the integration limit, $m=0.527697$, is found by solving (3.35) for $\theta=\pi / 4$. Numerical integration of (3.41) gives $c=0.48475$. This estimate of the circulation reduction is of the order of $\exp [(1 / 2 e \varepsilon)(e-1)]$ greater than that of MKO94. However, the circulation decay rate is still exponentially small and did not preclude us from finding the numerically steady solutions of $\S 2$.

This method of circulation decay rate calculation can be carried out for the generalized asymptotic solution (3.30). In general, the flux will be proportional to the vorticity on the dividing streamline and the length of the dividing streamline.

\subsubsection{Structure of vorticity leakage into Region IV}

To test the validity of our quasi-steady numerical results in the biaxial region, we used a time evolution code to examine how the vortices behave over time. It was 
found that the structure of the core of vortex persists over very long periods of time and that the effect of the biaxial strain is to strip away vorticity along a thin band on either side of the core, starting at the position of the stagnation point. This 'tail' of leaking vorticity was only present for strain ratios in the biaxial region and for at least moderate Reynolds numbers of order $10^{2}$ (see figure $2 c$ ). For lower circulation, biaxial strain will pull apart the vortex, but when the core of the vortex is close to circular, the tails appear.

We now establish a model for the structure of the tails. Working in a rectangular coordinate system $(\hat{x}, \hat{y})$ with origin at the stagnation point,

$$
\begin{aligned}
& \hat{x}=x-x_{s}, \\
& \hat{y}=y-y_{s},
\end{aligned}
$$

we assume that, in this region, the induced velocities of the vortex are negligible compared to the local strain. Hence, the vorticity equation becomes

$$
\frac{\partial \omega}{\partial t}-(1+\lambda) \hat{x} \frac{\partial \omega}{\partial \hat{x}}-(1-\lambda) \hat{y} \frac{\partial \omega}{\partial \hat{y}}=2 \omega+\frac{\partial^{2} \omega}{\partial \hat{x}^{2}}+\frac{\partial^{2} \omega}{\partial \hat{y}^{2}} .
$$

The solution of (3.44) subject to the boundary and initial conditions

$$
\begin{array}{lll}
\omega(\hat{x}, 0, t) & \text { given } & -\infty<\hat{x}<\infty, \\
\omega(\hat{x}, \hat{y}, 0) & \text { given } & -\infty<\hat{x}<\infty, 0<\hat{y}<\infty,
\end{array}
$$

is developed in Appendix B. We now assume that there is initially no vorticity in Region IV and so we set $\omega(\hat{x}, \hat{y}, 0)$, equal to zero in (3.46). At very large distances from the vortex, its detailed structure will not be resolvable. Hence in Region IV, we represent the leaking vorticity on the $\hat{x}$-axis in the form of a delta function with magnitude $A$, so that (3.45) is $\omega(\hat{x}, 0, t)=A \delta(\hat{x})$, where $A$ is to be determined. Using these initial and boundary conditions in the solution of Appendix B then gives, for the vorticity in Region IV

$$
\omega(\hat{x}, \hat{y}, t)=A \hat{y}\left(\frac{1}{\pi}\right)^{1 / 2} \int_{0}^{1} \frac{t \exp \left[-(1-\lambda) t \tau-\frac{1}{2} \hat{x}^{2} / P(\tau)-\frac{1}{2} \hat{y}^{2} / Q(\tau)\right]}{\left(-P(\tau) Q(\tau)^{3}\right)^{1 / 2}} \mathrm{~d} \tau,
$$

where

$$
\begin{aligned}
& P(\tau)=\frac{1-\mathrm{e}^{-2(1+\lambda) t \tau}}{1+\lambda}, \\
& Q(\tau)=\frac{1-\mathrm{e}^{-2(1-\lambda) t \tau}}{1-\lambda} .
\end{aligned}
$$

Taking the limit $t \rightarrow \infty$ of (3.47) gives the far-field vorticity

$$
\omega(\hat{x}, \hat{y}, t)=\left(\frac{1}{\pi}\right)^{1 / 2}\left(\frac{\lambda+1}{\lambda-1}\right)^{1 / 2} \frac{A}{\hat{y}} \mathrm{e}^{-(1+\lambda) \hat{x}^{2} / 2}+O\left(\frac{1}{t}\right),
$$

which is an approximation to the tail of leaking vorticity. This tail has a Gaussian cross-section with width $[2 /(1+\lambda)]^{1 / 2}$, which is equivalent to the width of a vortex sheet (2.5) embedded in the same strain field. Comparing this model to numerical results from a time advancing code, not shown here, indicated that this estimate of the width of the tail is very good. Table 7 and figure 9 show that, at $R=100, \lambda=1.5$, the tail has width corresponding to $2 /(1+\lambda)=0.8$.

To find the rate of circulation increase, the vorticity distribution (3.47) may be 


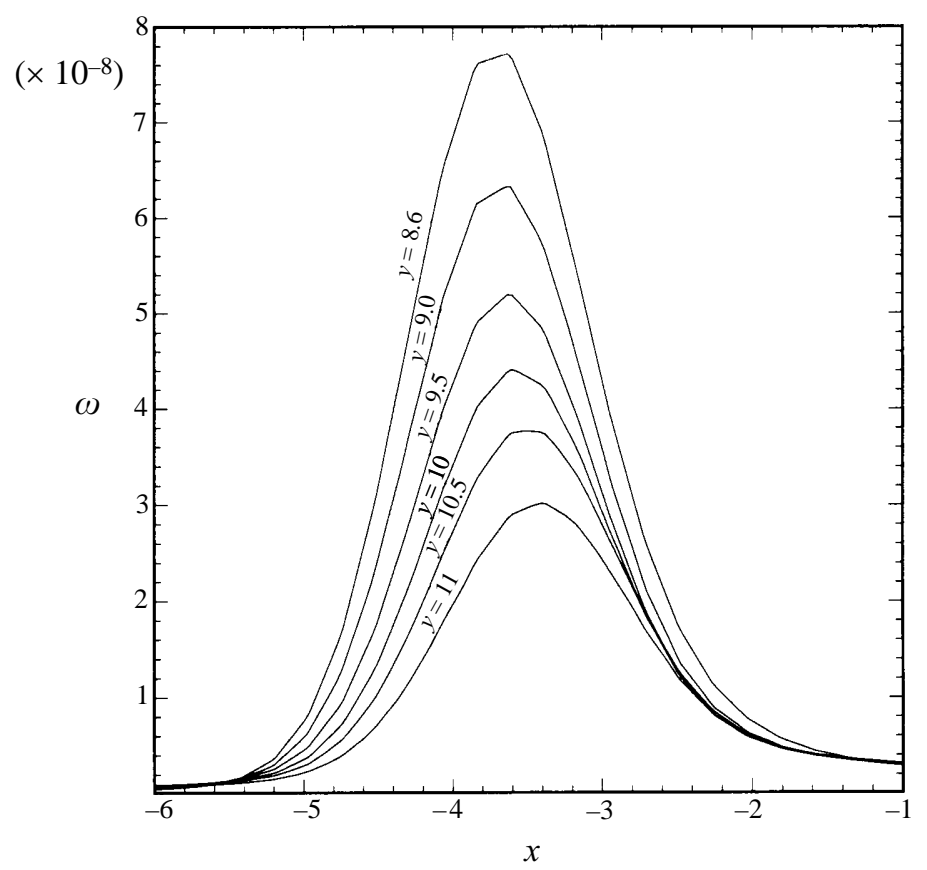

FIGURE 9. Cross-sections of 'tail' at various $y$ at $R=100, \lambda=1.5$.

$\begin{array}{ccc}x & y & \sigma^{2} \\ 3.70 & 8.6 & 0.77 \\ 3.67 & 9.0 & 0.75 \\ 3.63 & 9.5 & 0.77 \\ 3.57 & 10.0 & 0.79 \\ 3.50 & 10.5 & 0.79 \\ 3.43 & 11.0 & 0.79 \\ \text { TABLE 7. Results of curve-fitting a Gaussian, } \omega_{0} \mathrm{e}^{-\left(x-x_{s}\right)^{2} / \sigma^{2}} \text {, to the tail cross-sections of the } \\ \text { quasi-steady numerical solutions at } R=100, \lambda=1.5 \text {. Our analysis predicts } \sigma^{2}=0.8 .\end{array}$

integrated directly to give

$$
\frac{\partial \Gamma}{\partial t}=\frac{\partial}{\partial t} \int_{-\infty}^{\infty} \int_{0}^{\infty} \omega(\hat{x}, \hat{y}, t) \mathrm{d} \hat{x} \mathrm{~d} \hat{y}=\left(\frac{2(\lambda-1)}{\pi}\right)^{1 / 2} \frac{A}{\left(1-\mathrm{e}^{2(1-\lambda) t}\right)^{1 / 2}},
$$

which, as $t \rightarrow \infty$, limits to

$$
\frac{\partial \Gamma}{\partial t}=\left(\frac{2(\lambda-1)}{\pi}\right)^{1 / 2} A
$$

Matching this circulation increase to (3.40) requires that the magnitude of the delta function, $A$, be proportional to the cat's-eye boundary vorticity, $\sim \exp (1 / 2 e \varepsilon)$ and the length of the cat's-eye, $\sim \varepsilon^{-1 / 2}$, for large strain ratio, $\lambda=O\left(\varepsilon^{-1}\right)$.

These results indicate that the vorticity convected out of the cat's-eye develops into a tail of vorticity emanating from the stagnation points achieving a characteristic width equal to that of the Burgers vortex layer. Matching the vorticity leakage from 
one region to the other gives us confidence that our various approximations are physically reasonable.

\section{Two-dimensional stability of non-symmetric Burgers vortices}

We now consider the linear stability of the steady and quasi-steady solutions discussed in $\S 2$. We begin by demonstrating a property of general solutions of the vorticity equation for vorticity distributions in the presence of a linear background velocity field.

\subsection{General vorticity in a linear velocity field}

We return briefly to dimensional coordinates and consider a velocity field of the form

$$
u_{i}=\bar{u}_{i}\left(x_{i}, t\right)+J_{i j}(t) x_{j},
$$

where (4.1) represents a general velocity field $\bar{u}$ embedded in a linear background field. We take Cartesian coordinates $x_{i} \equiv\left(x_{1}, x_{2}, x_{3}\right)$ aligned with the non-rotating principal rates of strain with $\alpha(t)+\beta(t)+\gamma(t)=0$ and denote the background vorticity by $\xi_{i}$. The time-dependent rate-of-strain tensor of the background field is then

$$
\mathbf{J}(t)=\boldsymbol{S}(t)+\boldsymbol{A}(t)=\left[\begin{array}{ccc}
\alpha(t) & 0 & 0 \\
0 & \beta(t) & 0 \\
0 & 0 & \gamma(t)
\end{array}\right]+\left[\begin{array}{ccc}
0 & \frac{1}{2} \xi_{3}(t) & -\frac{1}{2} \xi_{2}(t) \\
-\frac{1}{2} \xi_{3}(t) & 0 & \frac{1}{2} \xi_{1}(t) \\
\frac{1}{2} \xi_{2}(t) & -\frac{1}{2} \xi_{1}(t) & 0
\end{array}\right] .
$$

The vorticity equation, then, in indicial notation is given by

$$
\frac{\partial \omega_{i}}{\partial t}+J_{j k} x_{k} \frac{\partial \omega_{i}}{\partial x_{j}}+\bar{u}_{j} \frac{\partial \omega_{i}}{\partial x_{j}}=\omega_{j} \frac{\partial \bar{u}_{i}}{\partial x_{j}}+J_{i j} \omega_{j}+v \nabla^{2} \omega_{i},
$$

where $\omega_{i}=\bar{\omega}_{i}+\xi_{i}=\epsilon_{i j k}\left(\partial u_{j} / \partial x_{k}\right)+\xi_{i}$. Making the change of variables to a system centred on a particle initially located at $x_{0_{i}}$ moving with the background flow

$$
x_{i}=X_{i}-x_{p_{i}}\left(t, x_{0_{i}}\right) \text {, }
$$

where

$$
\frac{\mathrm{d} x_{p_{i}}}{\mathrm{~d} t}=J_{i j} x_{p_{i}} \text { with } x_{p_{i}}(0)=x_{0_{i}}
$$

gives

$$
\frac{\partial \omega_{i}}{\partial t}+J_{j k}\left(X_{k}-x_{p_{k}}\right) \frac{\partial \omega_{i}}{\partial X_{j}}+\bar{u}_{j} \frac{\partial \omega_{i}}{\partial X_{j}}=\omega_{j} \frac{\partial \bar{u}_{i}}{\partial X_{j}}+J_{i j} \omega_{j}+v \nabla^{2} \omega_{i} .
$$

Defining transformed vorticity and velocity fields denoted by capitalized variables,

$$
\Omega_{i}\left(X_{i}, t\right)=\omega_{i}\left(X_{i}-x_{p_{i}}, t\right), \quad U_{i}\left(X_{i}, t\right)=\bar{u}_{i}\left(X_{i}-x_{p_{i}}, t\right),
$$

gives $\Omega$ which solves the vorticity equation

$$
\frac{\partial \Omega_{i}}{\partial t}+J_{j k} X_{k} \frac{\partial \Omega_{i}}{\partial X_{j}}+U_{j} \frac{\partial \Omega_{i}}{\partial X_{j}}=\Omega_{j} \frac{\partial U_{i}}{\partial X_{j}}+J_{i j} \Omega_{j}+v \nabla^{2} \Omega_{i},
$$

which is identical in form to (4.3). Note that the boundary conditions on this transformed equation may indeed be time dependent if the solution to (4.3) corresponds to velocities that do not decay to zero at infinity.

From the above we can conclude that for a given solution to (4.3), there exists an infinite family of additional solutions (indexed by $x_{0_{i}}$ ), each with the same form and evolution, corresponding to a displacement of the initial vorticity by $x_{0_{i}}$ and 
subsequent convection with the background flow. Alternatively we may say that solutions to (4.3) are invariant to an arbitrary initial displacement of the embedded vorticity field. We shall refer to this as the neutrally convective property of vorticity embedded in a linear field, and note that these results are also valid for the Euler and advection-diffusion equations. Furthermore, for the case of compact vorticity, we should note that the background vorticity $\xi_{i}$ must satisfy

$$
\frac{\partial \xi_{i}}{\partial t}=J_{i j} \xi_{j}=S_{i j} \xi_{j}
$$

yielding an alternative form for (4.3),

$$
\frac{\partial \bar{\omega}_{i}}{\partial t}+J_{j k} x_{k} \frac{\partial \bar{\omega}_{i}}{\partial x_{j}}+\bar{u}_{j} \frac{\partial \bar{\omega}_{i}}{\partial x_{j}}=\bar{\omega}_{j} \frac{\partial \bar{u}_{i}}{\partial x_{j}}+J_{i j} \bar{\omega}_{j}+S_{i j} \xi_{j}+v \nabla^{2} \omega_{i} .
$$

\subsection{A convectively neutral mode}

We now apply this result to our solutions of (4.3) corresponding to steady vorticity in the $z$-direction, where the background flow is a steady constant (in time) linear strain with no shear, $\xi \equiv 0$. Equations (4.4) and (4.7) become

$$
\left.\begin{array}{c}
x=X-x_{0} \mathrm{e}^{\alpha t}, \quad y=Y-y_{0} \mathrm{e}^{\beta t}, \\
\Omega(X, Y, t)=\omega\left(X-x_{0} \mathrm{e}^{\alpha t}, Y-y_{0} \mathrm{e}^{\beta t}\right), \\
U(X, Y, t)=\bar{u}\left(X-x_{0} \mathrm{e}^{\alpha t}, Y-y_{0} \mathrm{e}^{\beta t}\right), \\
V(X, Y, t)=\bar{v}\left(X-x_{0} \mathrm{e}^{\alpha t}, Y-y_{0} \mathrm{e}^{\beta t}\right),
\end{array}\right\}
$$

and we see that $\Omega(X, Y, t)$ is a convecting solution of (1.1) in the form

$$
\frac{\partial \Omega}{\partial t}+(\alpha X+U) \frac{\partial \Omega}{\partial X}+(\beta Y+V) \frac{\partial \Omega}{\partial Y}=\gamma \Omega+v \nabla^{2} \Omega .
$$

To relate this result to linear stability analysis, we write a general perturbed vorticity field in the form

$$
\omega(x, y, t)=\omega_{s s}(x, y)+\omega_{1}(x, y) \mathrm{e}^{-\mu t},
$$

where $\omega_{s s}$ is the steady base state and $\omega_{1},\left|\omega_{1}\right| \ll\left|\omega_{s s}\right|$, and $\mu$ are the eigenfunction and eigenvalue, respectively. Positive $\mu$ denotes linear stability and $\mu=0$ is the neutral stability boundary. Next we apply the neutrally convective property to $\omega_{s s}(x, y)$ and Taylor expand to obtain

$$
\omega_{s s}\left(x-x_{0} \mathrm{e}^{\alpha t}, y-y_{0} \mathrm{e}^{\beta t}\right) \simeq \omega_{s s}(x, y)-\frac{\partial \omega}{\partial x} x_{0} \mathrm{e}^{\alpha t}-\frac{\partial \omega}{\partial y} y_{0} \mathrm{e}^{\beta t} .
$$

Comparing the right-hand sides of (4.14)-(4.15) gives two linear eigensolutions $-(\partial \omega / \partial X) \mathrm{e}^{\alpha t}$ and $-(\partial \omega / \partial Y) \mathrm{e}^{\beta t}$ with corresponding eigenvalues $-\alpha$ and $-\beta$.

This result can be applied to various well-known solutions of the Navier-Stokes equations. For the Burgers vortex, the axisymmetric strain rates are $\alpha=\beta=-\gamma / 2$, so that, for any Reynolds number, this convective mode gives an eigenvalue equal to $\gamma / 2$. Given the non-dimensionalization in Prochazka \& Pullin (1995), this explains the appearance of stable eigenvalue $\mu=1$ for all Reynolds numbers. Secondly, for the Burgers vortex sheet solution (1.5) in plane strain, $\alpha=-\gamma, \beta=0$, we see that a perturbation in the $y$-direction is neutrally stable, $\mu=0$, but a displacement in $x$ results in a relaxation back to the base state. For the current study of non-symmetric Burgers vortices, upon non-dimensionalization and in terms of the strain ratio, the strain rates of the non-symmetric strain field result in eigenvalues of $\mu_{1}=1+\lambda$ and $\mu_{2}=1-\lambda$. Thus for $\lambda>1$, there exists at least one positive eigenvalue and therefore 
a linearly unstable mode. This does not correspond to a structural instability of the quasi-steady non-symmetric vortex but is merely the early-time linear phase of the nonlinear neutrally convective mode discussed previously.

\subsection{Subspace iteration}

In order to determine the stability boundary of the non-symmetric Burgers vortex in the $(R, \lambda)$-plane, we use a time advancing code in conjunction with a subspace iteration method to capture the fastest growing modes of the perturbation (Saad 1992). We restrict attention to two-dimensional perturbations, that is, those with velocity components normal to the vorticity. We now return to non-dimensional coordinates and write (2.1) in terms of non-dimensional cylindrical polar coordinate system $(r, \theta, z)$,

$$
\begin{gathered}
\frac{\partial \omega}{\partial t}=\frac{1}{r}\left[\frac{\partial}{\partial r}\left(r \frac{\partial \omega}{\partial r}\right)+\frac{1}{r} \frac{\partial^{2} \omega}{\partial \theta^{2}}\right]+\left[r(\lambda \cos 2 \theta+1)-R u_{r}\right] \frac{\partial \omega}{\partial r} \\
+\left[-\lambda \sin 2 \theta-\frac{R u_{\theta}}{r}\right] \frac{\partial \omega}{\partial \theta}+2 \omega, \\
\nabla^{2} \psi=-\omega, \quad u_{r}=\frac{1}{r} \frac{\partial \psi}{\partial \theta}, \quad u_{\theta}=-\frac{\partial \psi}{\partial r},
\end{gathered}
$$

where the velocity induced by the vorticity is now $\boldsymbol{u}=\left(u_{r}, u_{\theta}, 0\right)$. We linearize the equations with

$$
\omega=\omega+\omega^{\prime}, \quad u_{r}=u_{r_{0}}+u_{r}^{\prime}, \quad u_{\theta}=u_{\theta_{0}}+u_{\theta}^{\prime},
$$

where primed quantities are small perturbations, and we solve the resulting linear set using finite-difference methods in $r$ and spectral methods in $\theta$ (Buntine \& Pullin 1989). Taking the discrete Fourier transforms,

$$
\begin{aligned}
& \omega^{\prime}(r, \theta, t)=\mathrm{e}^{-\mu t} \sum_{n=-N / 2}^{N / 2-1} \hat{\omega}_{n}(r) \mathrm{e}^{\mathrm{i} n \theta}, \\
& \psi^{\prime}(r, \theta, t)=\mathrm{e}^{-\mu t} \sum_{n=-N / 2}^{N / 2-1} \hat{\psi}_{n}(r) \mathrm{e}^{\mathrm{i} n \theta},
\end{aligned}
$$

the Poisson equation becomes

$$
\frac{\mathrm{d}^{2} \hat{\psi}_{n}}{\mathrm{~d} r^{2}}+\frac{1}{r} \frac{\mathrm{d} \hat{\psi}_{n}}{\mathrm{~d} r}-\frac{n^{2}}{r^{2}} \hat{\psi}_{n}=-\hat{\omega}_{n}
$$

and the linearized vorticity equation becomes

$$
\begin{aligned}
\frac{\partial \hat{\omega}_{n}}{\partial t}= & \frac{-\lambda}{r}\left[\frac{\partial}{\partial r}\left(r^{2} \hat{\omega}_{n+2}\right)+\frac{\partial}{\partial r}\left(r^{2} \hat{\omega}_{n-2}\right)\right]-\lambda\left[(n+2) \hat{\omega}_{n+2}-(n-2) \hat{\omega}_{n-2}\right] \\
& +\frac{1}{r} \frac{\partial}{\partial r}\left(r^{2} \hat{\omega}_{n}\right)+\left[\frac{\partial^{2} \hat{\omega}_{n}}{\partial r^{2}}+\frac{1}{r} \frac{\partial \hat{\omega}_{n}}{\partial r}-\frac{n^{2}}{r^{2}} \hat{\omega}_{n}\right] \\
& -\mathscr{F}\left[u_{r_{0}} \frac{\partial \omega^{\prime}}{\partial r}+\frac{1}{r} u_{\theta_{0}} \frac{\partial \omega}{\partial \theta}+u_{r}^{\prime} \frac{\partial \omega_{0}}{\partial r}+\frac{1}{r} u_{\theta}^{\prime} \frac{\partial \omega_{0}}{\partial \theta}\right]
\end{aligned}
$$


for $N=-N / 2, \ldots, N / 2-1$ where $\mathscr{F}$ denotes the Fourier transform. The boundary conditions on (4.21)-(4.22) are

$$
\begin{aligned}
& r=0: \hat{\psi}_{n}=0, \quad \hat{\omega}_{n}=0, n \neq 0, \quad \frac{\mathrm{d} \hat{\omega}_{0}}{\mathrm{~d} r}=0, \\
& r \rightarrow \infty: \hat{\psi}_{n}(r) \rightarrow 0, \quad \hat{\omega}_{n}(r) \rightarrow 0 .
\end{aligned}
$$

Equation (4.22) is solved by a fourth-order five-point spatial finite-difference scheme. The system is advanced in time first with a second-order explicit predictor followed by updating the vorticity in a Crank-Nicolson semi-implicit two-point corrector scheme. This updated vorticity $\hat{\omega}(t+\delta t)$ is then used in the Poisson solver. Several iterations of this procedure are needed per time step for convergence. Further details of the scheme are given in Buntine \& Pullin (1989). All calculations reported here use $N=256$ in (4.19)-(4.20). Initial conditions are obtained from the steady and quasi-steady solutions of $\S 2$ by projection of the values of $\omega$ and $\psi$ onto the $r, \theta$ grid.

Initially, a simple power method approach is used in which, if we represent the time advancement as the function $A$, a random initial guess, $\hat{\omega}$, is iterated on $A$, i.e.

$$
\hat{\omega}_{k+1}=\frac{1}{\left|\hat{\omega}_{k+1}\right|} A \hat{\omega}_{k},
$$

where $k$ denotes the iteration number and $\left|\hat{\omega}_{k+1}\right|$ is the $L^{2}$ norm of the vector $A \hat{\omega}_{k}$. The eigenvalue is then calculated using the Rayleigh quotient,

$$
\mu_{k}=\frac{\left\langle A \omega_{k}, \omega_{k}\right\rangle}{\left\langle\omega_{k}, \omega_{k}\right\rangle} .
$$

Iteration is continued until $\left|\mu_{k}-\mu_{k-1}\right|<10^{-3}$. Maximum eigenvalues are found using this method for $R=1,10,100$, and 1000 and are shown in tables 8 and 9 . From these results it is seen that the eigenvalue corresponding to the fastest growing eigenfunction corresponds closely to $\mu=1-\lambda$. The corresponding numerically obtained eigenfunctions are also found to be closely proportional to $\partial \omega_{s s} / \partial y$. We may thus conclude that the mode with maximum growth rate corresponds to a neutrally convective translation of the non-symmetric vortex without change of shape and that this convection is toward the origin for $\lambda<1$. Therefore, all eigenvalues are positive for $\lambda<1$ and the non-symmetric Burgers vortex is linearly stable in this region, at least for the values of $R$ investigated.

In the region $\lambda>1$, these eigenvalues are positive, allowing the possibility that other eigenfunctions exist with $0<\mu<1-\lambda$ that are not convectively neutral. These eigenfunctions are examined by employing a multi-step subspace iteration technique (Saad 1992) in which we iterate on the three-vector system $W_{k}=\left(\hat{\omega}_{1}, \hat{\omega}_{2}, \hat{\omega}_{3}\right)$, where $\hat{\omega}_{1}$ and $\hat{\omega}_{2}$ are equal to the analytical form of the convectively neutral eigenfunctions and $\hat{\omega}_{3}$ is a random vector. We calculate

$$
V_{k+1}=A^{p} W_{k}
$$

and compute the $Q R$ factorization $V_{k+1}=Q R$ to find $W_{k+1}=Q$. Since the cost of this orthonormalization can be high, it is only performed after $p$ iterations on $A$. Computing the Rayleigh quotients yield the two analytically predicted eigenvalues $1 \pm \lambda$ and a third eigenvalue which corresponds to the fastest growing eigenfunction not found previously. This eigenfunction consistently corresponds to an eigenvalue approximately equal to zero within our numerical accuracy for all Reynolds numbers studied. We hypothesize that this eigenmode represents the exceedingly slow evolution 


$\begin{array}{ccc}\lambda & \mu_{\max }, R=1 & \mu_{\max }, R=10 \\ 0.2 & 0.8168 & 0.8014 \\ 0.4 & 0.6093 & 0.6050 \\ 0.6 & 0.4040 & 0.4033 \\ 0.8 & 0.2058 & 0.2046\end{array}$

TABLE 8. Maximum eigenvalue for $R=1,10$.

$\begin{array}{ccc}\lambda & \mu_{\max }, R=100 & \mu_{\max }, R=1000 \\ 0.5 & 0.5056 & - \\ 1.0 & 0.0203 & 0.0126 \\ 1.5 & -0.5031 & - \\ 2.0 & - & -0.9956 \\ 3.0 & - & -1.9965\end{array}$

TABLE 9. Maximum eigenvalue for $R=100,1000$.

of the quasi-steady numerical solutions for non-symmetric Burgers vortices with $\lambda>1$. We subsequently argue that this comprises leakage of vorticity occurring near stagnation points of the steady flow. A model for this leakage was discussed in $\S 3$.

\subsection{Results}

The linear stability analysis has analytically shown that at least one positive eigenvalue exists for $\lambda>1$. Our result shows that in a biaxial strain field, a general small perturbation to the vortex will result in the vortex moving with the strain field.

For the range of Reynolds numbers investigated, $R=1,10,100,1000$, we find that the two smallest (most negative) eigenvalues in fact do correspond to the translating analytical solutions found in the previous section for $\lambda<1$. For $\lambda>1$, the smallest eigenvalue, $1-\lambda$, corresponds to the convectively neutral mode and the second smallest eigenvalue, $\approx 0$, corresponds to the slow leakage of vorticity into tails.

\section{Summary}

The steady and quasi-steady structure of non-symmetric Burgers vortices was calculated numerically for a wide range of strain ratios $(0.2<\lambda<150)$ and Reynolds numbers $\left(1<R<10^{4}\right)$, which includes strain ratios well into the biaxial region, $\lambda>1$. An asymptotic formulation for the analytic form of the vorticity for large $R$ and $\lambda$ but $\varepsilon \ll 1$, in the region outside of the core of the vorticity but within the cat's-eye boundary, was found to be

$$
\omega_{I I}(r, \theta)=\mathrm{e}^{-r^{2} \exp \left(\varepsilon r^{2} \sin 2 \theta\right) / 2},
$$

The non-symmetric Burgers vortex was found to be linearly stable for $\lambda<1$. When $\lambda \geqslant 1$, an apparent normal-mode instability corresponds to a neutrally convective mode where the vortex moves with the background strain without change of structure. An analytic explanation for this mode has been presented.

When $\lambda>1$, our estimate for the flux of vorticity out of the cat's-eye and decay of circulation was found to be exponentially small, given in dimensional variables,

$$
\frac{\partial \Gamma}{\partial t}=\frac{0.48475}{4 \pi} \gamma \varepsilon^{-1} \Gamma \exp \left(\frac{-1}{2 e \varepsilon}\right) .
$$


The structure of this leaking vorticity was found to be in the form of 'tails' emanating from the stagnation points of the flow whose long-time behaviour closely resembled a time-dependent Burgers vortex layer.

In conclusion, the generalized Burgers vortex is effectively stable to two-dimensional perturbations. Its stability to perturbations containing generalized velocity disturbances in the axial direction remains an open problem.

\section{Appendix A. Streamfunction correction}

Here we show that the terms of (3.19) are the products of quantities that are themselves exponentially small in Region II, so that the terms of $L_{4}$ are indeed small compared to those of $L_{1}$ and $L_{3}$. It is sufficient to calculate $\psi_{1}$, the streamfunction associated with $\omega_{I I}$. From (3.13)-(3.15) and (3.22)

$$
\nabla^{2} \psi_{1}=-\omega_{I I}=-\mathrm{e}^{-r^{2} \exp \left(\varepsilon r^{2} \sin 2 \theta\right) / 2}
$$

which was solved by first writing

$$
\psi_{1}=\mathrm{e}^{-r^{2} \exp \left(\varepsilon r^{2} \sin 2 \theta\right) / 2}\left[f_{1}(r)+\varepsilon f_{2}(r) \sin 2 \theta+\cdots\right] .
$$

The resulting equation for $f_{1}$ is

$$
f_{1}^{\prime \prime}+\left(\frac{1}{r}-2 r\right) f_{1}^{\prime}+\left(r^{2}-1\right) f_{1}=1,
$$

which is solved using variation of parameters of the homogeneous solutions, $\exp \left(-r^{2} / 2\right), \ln r \exp \left(-r^{2} / 2\right)$, and we find,

$$
f_{1}(r)=-\frac{1}{2} \mathrm{E}_{1}\left(\frac{r^{2}}{2}\right) \mathrm{e}^{r^{2} / 2}
$$

where $E_{1}$ is the exponential integral, as defined by Abromowitz \& Stegun (1972),

$$
\mathrm{E}_{1}(z)=\int_{z}^{\infty} \frac{\mathrm{e}^{-t}}{t} \mathrm{~d} t
$$

Then, (A 4) forces the equation for $f_{2}$,

$$
f_{2}^{\prime \prime}+\left(\frac{1}{r}-2 r\right) f_{2}^{\prime}+\left(r^{2}-\frac{4}{r^{2}}-2\right) f_{2}=4 r^{2}+3 r^{2} \mathrm{E}_{1}\left(\frac{-r^{2}}{2}\right) \mathrm{e}^{r^{2} / 2},
$$

which is also solved by various parameters of the homogeneous solutions, $r^{ \pm 2} \exp \left(r^{2} / 2\right)$. Then, using the boundary condition that $f_{2} \rightarrow 0$ as $r \rightarrow 0$, we find

$$
f_{2}(r, \theta)=\frac{r^{4}}{4} \mathrm{E}_{1}\left(\frac{r^{2}}{2}\right) \mathrm{e}^{r^{2} / 2} \sin 2 \theta+\frac{6 \sin 2 \theta}{r^{2}}\left(1+\frac{r^{2}}{2}+\frac{r^{4}}{12}-\mathrm{e}^{-r^{2} / 2}\right) .
$$

Substituting (A 4) and (A 7) into (A 1), using properties of the exponential integral, and matching to the Burgers vortex as $r \rightarrow 0$ yields

$$
\psi_{1}=\frac{1}{2} \mathrm{E}_{1}\left(\frac{-r^{2}}{2}\right)+\varepsilon \frac{6 \sin 2 \theta}{r^{2}}\left(1+\frac{r^{2}}{2}+\frac{r^{4}}{12}-\mathrm{e}^{-r^{2} / 2}\right) \mathrm{e}^{-r^{2} \exp \left(r r^{2} \sin 2 \theta\right) / 2}+O\left(\varepsilon^{2}\right) .
$$

Thus, the corrections to the streamfunction in Region II are exponentially small and therefore the terms of $L_{4}$ are small compared to $L_{1}$, and both are exponentially smaller than the terms of $L_{3}$. 
Using (A8), the streamfunction in Region II may be represented as

$$
\begin{aligned}
\Psi= & -\int_{0}^{r}\left(1-\mathrm{e}^{-\rho^{2} / 2}\right) \frac{1}{\rho} \mathrm{d} \rho-\varepsilon r^{2} \sin 2 \theta \\
& +\varepsilon \frac{6 \sin 2 \theta}{r^{2}}\left(1+\frac{r^{2}}{2}+\frac{r^{4}}{12}-\mathrm{e}^{-r^{2} / 2}\right) \mathrm{e}^{-r^{2} \exp \left(\varepsilon r^{2} \sin 2 \theta\right) / 2}+O\left(\varepsilon^{2}\right),
\end{aligned}
$$

where we have replaced the $-\ln r$ term by the exact streamfunction for the Burgers vortex.

\section{Appendix B. The convection-diffusion equation}

Here we solve the convection-diffusion equation (3.44) subject to arbitrary initial and boundary conditions. First, introducing the transformation

$$
X=\hat{x} \mathrm{e}^{(1+\lambda) t}, \quad Y=\hat{y} \mathrm{e}^{(1-\lambda) t}, \quad \Omega(X, Y, t)=\omega\left(X \mathrm{e}^{-(1+\lambda) t}, Y \mathrm{e}^{-(1-\lambda) t}, t\right),
$$

yields

$$
\frac{\partial \Omega}{\partial t}=2 \Omega+\mathrm{e}^{2(1+\lambda) t} \frac{\partial^{2} \Omega}{\partial X^{2}}+\mathrm{e}^{2(1-\lambda) t} \frac{\partial^{2} \Omega}{\partial Y^{2}},
$$

with initial distribution and boundary conditions

$$
\begin{aligned}
& \omega\left(X \mathrm{e}^{-(1+\lambda) t}, 0, t\right)=\Omega(X, 0, t),-\infty<x<\infty, \\
& \quad \omega(x, y, 0)=\Omega(X, Y, 0),-\infty<x<\infty, 0<y<\infty .
\end{aligned}
$$

Taking the Fourier transform in $X$,

$$
\widetilde{\Omega}\left(k_{1}, Y, t\right)=\frac{1}{2 \pi} \int_{-\infty}^{\infty} \Omega \mathrm{e}^{\mathrm{i} k_{1} X} \mathrm{~d} X, \quad \Omega(X, Y, t)=\int_{-\infty}^{\infty} \widetilde{\Omega} \mathrm{e}^{-\mathrm{i} k_{1} X} \mathrm{~d} k_{1},
$$

and then taking the Fourier sine transform in $\mathrm{Y}$,

$$
\hat{\Omega}\left(k_{1}, k_{2}, t\right)=\frac{2}{\pi} \int_{0}^{\infty} \widetilde{\Omega} \sin \left(k_{2} Y\right) \mathrm{d} Y, \quad \widetilde{\Omega}\left(k_{1}, Y, t\right)=\int_{0}^{\infty} \hat{\Omega} \sin \left(k_{2} Y\right) \mathrm{d} k_{2},
$$

the equation becomes

$$
\frac{\partial \hat{\Omega}}{\partial t}=\hat{\Omega}\left(2-k_{1}^{2} \mathrm{e}^{2(1+\lambda) t}-k_{2}^{2} \mathrm{e}^{2(1-\lambda) t}\right)+\frac{2}{\pi} k_{2} \mathrm{e}^{2(1-\lambda) t} \widetilde{\Omega}\left(k_{1}, 0, t\right) .
$$

Writing this in terms of a derivative prefactor, $F(k 1, k 2, t)$,

$$
\begin{aligned}
\frac{\mathrm{d}}{\mathrm{d} t}\left[F(k 1, k 2, t) \hat{\Omega}\left(k_{1}, k_{2}, t\right)\right] & =\frac{2}{\pi} k_{2} \mathrm{e}^{2(1-\lambda) t} F(k 1, k 2, t) \widetilde{\Omega}\left(k_{1}, 0, t\right), \\
F(k 1, k 2, t) & =\mathrm{e}^{-2 t} G_{1}(\lambda, t) G_{2}(\lambda, t), \\
G_{n}(\lambda, t) & =\exp \left[\frac{k_{n}^{2} \mathrm{e}^{2\left(1-(-1)^{n} \lambda\right) t}}{2\left(1-(-1)^{n} \lambda\right)}\right] .
\end{aligned}
$$

Upon integrating over time and solving for $\hat{\Omega}\left(k_{1}, k_{2}, t\right)$, we find

$$
\begin{aligned}
\hat{\Omega}\left(k_{1}, k_{2}, t\right)= & \hat{\Omega}\left(k_{1}, k_{2}, 0\right) \exp [2 t] \frac{G_{1}(\lambda, 0)}{G_{1}(\lambda, t)} \frac{G_{2}(\lambda, 0)}{G_{2}(\lambda, t)} \\
& +\frac{2}{\pi} k_{2} \int_{0}^{t} \widetilde{\Omega}\left(k_{1}, 0, \tau\right) \exp [2 t-2 \lambda \tau] \frac{G_{1}(\lambda, \tau)}{G_{1}(\lambda, t)} \frac{G_{2}(\lambda, \tau)}{G_{2}(\lambda, t)} \mathrm{d} \tau .
\end{aligned}
$$


Transforming the vorticity back into physical space yields

$$
\begin{aligned}
\Omega(X, Y, t)= & \mathrm{e}^{2 t} \int_{-\infty}^{\infty} \int_{0}^{\infty} \hat{\Omega}\left(k_{1}, k_{2}, 0\right) \frac{G_{1}(\lambda, 0)}{G_{1}(\lambda, t)} \frac{G_{2}(\lambda, 0)}{G_{2}(\lambda, t)} \mathrm{e}^{-\mathrm{i} k_{1} X} \sin \left(k_{2} Y\right) \mathrm{d} k_{1} \mathrm{~d} k_{2} \\
& +\left(\frac{2}{\pi}\right)^{1 / 2} \int_{0}^{t} \mathrm{e}^{2 t-2 \lambda \tau} \frac{(\lambda-1)^{3 / 2} Y \exp \left[\frac{(1-\lambda) Y^{2} / 2}{\mathrm{e}^{2(1-\lambda) \tau}-\mathrm{e}^{2(1-\lambda) t}}\right]}{\left(\mathrm{e}^{2(1-\lambda) t}-\mathrm{e}^{2(1-\lambda) \tau}\right)^{3 / 2}} \\
& \times \int_{-\infty}^{\infty} \widetilde{\Omega}\left(k_{1}, 0, \tau\right) \frac{G_{1}(\lambda, \tau)}{G_{1}(\lambda, t)} \mathrm{e}^{-\mathrm{i} k_{1} X} \mathrm{~d} k_{1} \mathrm{~d} \tau .
\end{aligned}
$$

We gratefully acknowledge helpful discussions with David Hill, James Buntine, and Ron Henderson. This research was partial supported by NSF Grant CTS-9634222.

\section{REFERENCES}

Abramowitz, M. \& Stegun, I. A. 1972 Handbook of Mathematical Functions. Nat. Bur. Standards; Dover.

Ashurst, W. T., Kerstein, A. R., Kerr, R. M. \& Gibson. C. H. 1987 Alignment of vorticity and scalar gradient with strain in simulated Navier-Stokes turbulence. Phys. Fluids 30, 2343-2353.

BAJER, K. \& Moffatt. H. K. 1997 On the effect of a central vortex on a stretched magnetic-flux tube. J. Fluid Mech. 339, 121-142.

Berry, M. 1991 Asymptotics, superasymptotics, hyperasymptotics. In Asymptotics Beyond all Orders (ed. H. Segur, S. Tanveer \& H. Levine). Plenum.

Buntine, J. D. \& Pullin, D. I. 1989 Merger and cancellation of strained vortices. J. Fluid Mech. 205, 263-295.

Burgers, J. M. 1948 A mathematical model illustrating the theory of turbulence. Adv. Appl. Mech. 1, 171-199.

Douady, S. Couder, Y. \& Brachet, M. E. 1991 Direct observation of the intermittency of intense vorticity filaments in turbulence. Phys. Rev. Lett. 67, 983-986.

JimÉnez, J., Moffatt, H. K. \& VASCO, C. 1996 The structure of vortices in freely decaying twodimensional turbulence. J. Fluid Mech. 313, 209-222.

JimÉNEZ, J. \& WrAY, A. A. 1994 Columnar vortices in isotropic turbulence. Meccanica 29, 453-464.

Jiménez, J., Wray, A. A., Saffman, P. G. \& Rogallo, R. S. 1993 The structure of intense vorticity in isotropic turbulence. J. Fluid Mech. 255, 65-90.

KERR, R. M. 1985 Higher order derivative correlation and the alignment of small-scale structures in isotropic turbulence. J. Fluid Mech. 153, 31-58.

KIDA, S. \& OHKITANI, K. 1992 Spatiotemporal intermittency and instability of a forced turbulence. Phys. Fluids A 4, 1018-1027.

Leibovich, S. \& Holmes, P. 1981 Global stability of Burgers vortex. Phys. Fluids 24, 548-549.

Lin, S. J. \& CoRcos, G. M. 1984 The mixing layer: deterministic models of a turbulent flow. Part 3. the effect of plane strain on the dynamics of streamwise vortices. J. Fluid Mech. 141, 139-178.

LundGREN, T. S. 1982 Strained spiral vortex model for turbulent fine structure. Phys. Fluids 25, 2193-2203.

Moffatt, H. K., Kida, H. K. \& Ohkitani, K. 1994 Stretched vortices - the sinews of turbulence; large-Reynolds-number asymptotics. J. Fluid Mech. 259, 241-264 (referred to herein as MK094).

ProchazKa, A. \& Pullin, D. I. 1995 On the two-dimensional stability of the axisymmetric Burgers vortex. Phys. Fluids 7, 1788-1790.

Reutsch, G. R. \& MaXey, M. A. 1991 Small scale features of vorticity and passive scalar fields in homogeneous isotropic turbulence. Phys. Fluids A 3, 1587-1597.

Robinson, A. C. \& Saffman, P. G. 1984. Stability and structure of stretched vortices. Stud. Appl. Maths 70, 163-181 (referred to herein as RS84).

SAAD, Y. 1992 Numerical Methods for Large Eigenvalue Problems. Manchester University Press. 
Saffman, P. G. \& Pullin, D. I. 1996 Calculation of velocity structure functions for vortex models of isotropic turbulence. Phys. Fluids 8, 3072-3084.

She, Z.-S., JACKsOn, E. \& OrszaG, S. A. 1991 Structure and dynamics of homogeneous turbulence - models and simulations. Proc. R. Soc. Lond. A 434, 101-124.

TAYlor, G. I. 1938 Production and dissipation of vorticity in a turbulent fluid. Proc. R. Soc. Lond. A 164, 15-23.

Townsend, A. A. 1951 On the fine-scale structure of turbulence. Proc. R. Soc. Lond. A 208, 534-542.

Vincent, A. \& MeneguzzI, M. 1991 The spatial and statistical properties of homogeneous turbulence. J. Fluid Mech. 225, 1-20. 